\title{
$\mathbf{J}|\mathbf{A}| \mathbf{C} \mid \mathbf{S}$
}

ARTICLES

\section{Towards an Improved Understanding of the Glutamate Mutase System}

\author{
Gregory M. Sandala, ${ }^{\dagger, \diamond, \pi}$ David M. Smith,", E. Neil G. Marsh, ${ }^{\S}$ \\ and Leo Radom $+, \diamond, \pi, *$
}

School of Chemistry and ARC Centre of Excellence in Free Radical Chemistry and Biotechnology, University of Sydney, Sydney, NSW 2006, Australia, Research School of Chemistry, Australian National University, Canberra, ACT 0200, Australia, Rudjer Boskovic Institute, 10002 Zagreb,

Croatia, and Department of Chemistry, University of Michigan, Ann

Arbor, MI 48109-1055, USA

\section{SUPPORTING INFORMATION}

(Total 26 pages including this page)

\footnotetext{
${ }^{\dagger}$ University of Sydney

$\checkmark$ Australian National University

- Rudjer Boskovic Institute

${ }^{\S}$ University of Michigan

"ARC Centre of Excellence in Free Radical Chemistry and Biotechnology

*E-mail: david.smith@irb.hr; radom@chem.usyd.edu.au
} 


\section{Hypothetical Isomerization of the Substrate Analogue 2,2-Diaminoglutaric Acid}

The radical stabilization energies for the various substituted analogues of glycyl radical (Table 1) potentially allow us to predict beforehand which fragmentation products in a series of GM-catalyzed reactions are likely to be of higher energy and which are of lower energy. To illustrate the possible benefits of such an exercise, we have computed the energy requirements for the hypothetical isomerization of 2,2-diaminoglutaric acid (1s) to 2,2-diamino-3-methylsuccinic acid (5s) in a manner that is consistent with the catalytic pathway for GM (Scheme 2). The fragmentation step in this process involves the 2aminoglycyl radical, $\mathrm{H}_{2} \mathrm{~N}-\bullet \mathrm{C}\left(\mathrm{CO}_{2} \mathrm{H}\right)-\mathrm{NH}_{2}(3 \mathrm{~s}$, Table 1$)$, which we have found to be a strongly stabilized radical.

Scheme S1 shows the initial hydrogen abstraction by Ado from 2,2-diaminoglutaric acid (1s) to form the substrate-derived radical $2 \mathrm{~s}$ to be exothermic by $39.5 \mathrm{~kJ} \mathrm{~mol}^{-1}$. The barrier for fragmentation of the substrate-derived radical $2 \mathrm{~s}$ is calculated to be $48.7 \mathrm{~kJ}$ $\mathrm{mol}^{-1}$, and the process is endothermic by only $26.3 \mathrm{~kJ} \mathrm{~mol}^{-1}$. 
Scheme S1. Schematic Energy Profile for the Hypothetical Conversion of 2,2Diaminoglutaric Acid (1s) to 2,2-Diamino-3-Methylsuccinic Acid (5s) Catalyzed by Glutamate Mutase (energies relative to $1 \mathrm{~s}$ are given in parentheses, $\mathrm{kJ} \mathrm{mol}^{-1}$ )

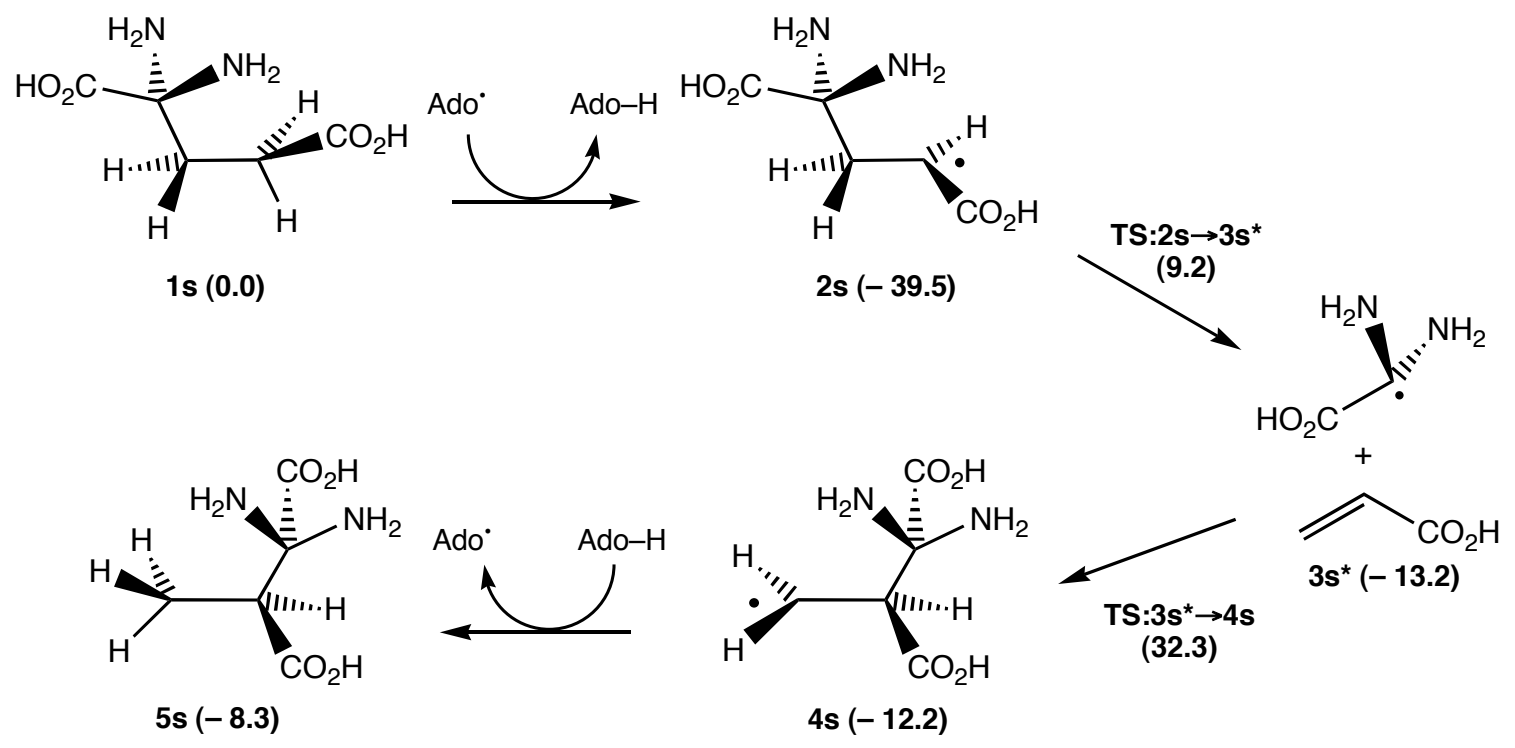

Recombination of the fragments comprising $3 \mathrm{~s}^{*}$ (with a barrier of $45.5 \mathrm{~kJ} \mathrm{~mol}^{-1}$ ) yields the product-related radical $4 \mathrm{~s}$ in a reaction that is endothermic by $1.0 \mathrm{~kJ} \mathrm{~mol}^{-1}$, leaving $4 \mathrm{~s}$ $27.4 \mathrm{~kJ} \mathrm{~mol}^{-1}$ higher in energy than the substrate-derived radical 2s. Finally, we calculate that hydrogen re-abstraction by $\mathbf{4} \mathbf{s}$ from Ado-H to generate the product 2,2-diamino-3methylsuccinic acid (5s) and re-form Ado is slightly endothermic.

At this point, it is instructive to compare the results shown for the isomerization of 2,2diaminoglutaric acid (1s) with those for $(S)$-glutamic acid (1a). In this context, it is pertinent to recall the data in Table 1, which show the RSE for $3 \mathrm{~s}$ to be $20.5 \mathrm{~kJ} \mathrm{~mol}^{-1}$ greater than that of glycyl radical (3a). A higher RSE for a particular radical might be expected to lead to lower energy demands for reactions involving that radical compared with those involving a radical with a lower RSE. Indeed, we observe the fragmentation barrier for $2 \mathbf{s}$ to be $16.0 \mathrm{~kJ} \mathrm{~mol}^{-1}$ lower than that for $\mathbf{2 a}$ (Scheme 2). Similarly, the 
fragmentation enthalpy of $2 \mathrm{~s}$ is $11.7 \mathrm{~kJ} \mathrm{~mol}^{-1}$ lower than that for the $\mathbf{2 a}$. Furthermore, we observe a similar trend, albeit to a smaller degree, with the reverse fragmentation barrier of $4 s$.

On the basis of a $20.5 \mathrm{~kJ} \mathrm{~mol}^{-1}$ difference in RSEs between $3 \mathbf{s}$ and 3a, one might expect larger energy differences along their respective reaction pathways than is actually observed in the explicit calculations. However, the relative stabilities of the substratederived and product-related radicals also contribute to the energy profile, and their contributions may not be trivial. Such a scenario is exemplified with $2 \mathbf{s}$ and $4 \mathbf{s}$ where intramolecular hydrogen bonds artificially stabilize these species. These artifacts of our truncated gas-phase models might potentially cancel in the enzyme environment. Hence, the use of RSEs to provide a qualitative indication of the propensity for the fragmentation process retains merit. 


\section{GAUSSIAN Archive Entries for the B3-LYP/6-31G(d,p) Optimized Geometries}

$1 \mathbf{a}$

$1 \backslash 1 \backslash G I N C-A C 18 \backslash S P \backslash R O M P 2-F C \backslash 6-31 G(d) \backslash C 5 H 9 N 104 \backslash G M S 501 \backslash 12-S e p-2005 \backslash 0 \backslash \backslash \# R O M$ P2/6-31G* SCF=TIGHT \Iglutamate from substrate radical conformer 1 b3ly $\mathrm{p} / 6-31 \mathrm{G} * * \backslash \backslash 0,1 \backslash \mathrm{C}, 0,0.017296,-0.888529,-0.369755 \backslash \mathrm{C}, 0,0.945465,-0.013465$ $, 0.490802 \backslash \mathrm{N}, 0,0.627195,1.427103,0.478173 \backslash \mathrm{H}, 0,-0.196326,1.61678,-0.0899$ $25 \backslash \mathrm{H}, 0,0.44945,1.782766,1.411291 \backslash \mathrm{C}, 0,-1.405502,-1.089508,0.166793 \backslash \mathrm{C}, 0$, $-2.376322,0.03376,-0.126292 \backslash 0,0,-3.633128,-0.292672,0.241185 \backslash \mathrm{H}, 0,-4.19$ $4187,0.470389,0.020157 \backslash 0,0,-2.113674,1.107116,-0.635076 \backslash \mathrm{H}, 0,0.503076,-$ $1.864384,-0.446416 \backslash \mathrm{H}, 0,-0.027524,-0.476794,-1.38506 \backslash \mathrm{H}, 0,0.928165,-0.40$ $1443,1.517987 \backslash \mathrm{C}, 0,2.406519,-0.211702,0.00365 \backslash 0,0,3.021258,0.932249,-0$. $301195 \backslash 0,0,2.920207,-1.305034,-0.082504 \backslash \mathrm{H}, 0,-1.406874,-1.254763,1.2523$ $\backslash \mathrm{H}, 0,2.318718,1.619018,-0.1384 \backslash \mathrm{H}, 0,-1.846899,-1.997885,-0.259608 \backslash \backslash \mathrm{Vers}$ ion $=I A 64 L-G 03 R e v C .02 \backslash$ State $=1-A \backslash H F=-548.5089535 \backslash M P 2=-550.028034 \backslash R M S D=4$. $654 e-09 \backslash P G=C 01 \quad[X(C 5 H 9 N 104)] \backslash \backslash @$

\section{2a}

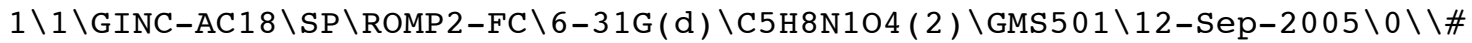
ROMP 2/6-31G* SCF=TIGHT\\glutamate from substrate radical conformer $1 \mathrm{~b}$ $31 \mathrm{yp} / 6-31 \mathrm{G} * * \backslash \backslash 0,2 \backslash \mathrm{C}, 0,-0.008129,-0.910072,-0.35975 \backslash \mathrm{C}, 0,0.93037,-0.0265$ $35,0.513878 \backslash \mathrm{N}, 0,0.587308,1.40351,0.507141 \backslash \mathrm{H}, 0,-0.189106,1.592641,-0.12$ $64 \backslash \mathrm{H}, 0,0.313168,1.729499,1.427643 \backslash \mathrm{C}, 0,-1.392205,-1.024566,0.168259 \backslash \mathrm{C}, 0$ $,-2.388663,-0.021016,-0.139034 \backslash 0,0,-3.626553,-0.333607,0.318057 \backslash \mathrm{H}, 0,-4$ $.205904,0.40233,0.059751 \backslash 0,0,-2.164233,1.026193,-0.745207 \backslash \mathrm{H}, 0,0.4642,-$ $1.893409,-0.425213 \backslash \mathrm{H}, 0,-0.031313,-0.472488,-1.365132 \backslash \mathrm{H}, 0,0.924457,-0.4$ $15649,1.538925 \backslash \mathrm{C}, 0,2.38509,-0.213394,0.001989 \backslash 0,0,3.005639,0.940169,-0$ $.247945 \backslash \mathrm{O}, 0,2.887433,-1.304728,-0.142294 \backslash \mathrm{H}, 0,-1.675742,-1.843905,0.821$ $238 \backslash \mathrm{H}, 0,2.31203,1.625697,-0.053731 \backslash \backslash$ Version=IA64L-G03RevC . 02 $\backslash$ State $=2-\mathrm{A}$ $\backslash \mathrm{HF}=-547.8836873 \backslash \mathrm{MP} 2=-549.3824826 \backslash \mathrm{RMSD}=4.055 \mathrm{e}-09 \backslash \mathrm{PG}=\mathrm{C} 01 \quad[\mathrm{X}(\mathrm{C} 5 \mathrm{H} 8 \mathrm{~N} 104)] \backslash$ $\backslash$ @

\section{C3-radical derived from 1a}

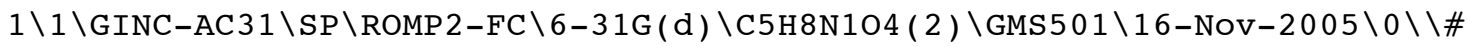
ROMP 2/6-31G* SCF=TIGHT \\C3 radical glutamate coords from 1I9C b31yp/6$31 \mathrm{G}(\mathrm{d}, \mathrm{p}) \backslash \backslash 0,2 \backslash \mathrm{N}, 0,2.012345,-1.773209,-0.222656 \backslash \mathrm{C}, 0,1.053292,-0.674493$, $-0.229603 \backslash \mathrm{C}, 0,1.744026,0.696901,-0.101655 \backslash 0,0,2.890025,0.864935,0.2512$ $18 \backslash \mathrm{C}, 0,0.045771,-0.858174,0.874607 \backslash \mathrm{C}, 0,-1.168141,-0.006633,1.0414 \backslash \mathrm{C}, 0$, $-2.162553,-0.089493,-0.11439 \backslash 0,0,-1.958033,-0.556262,-1.212518 \backslash 0,0,-3$. $347724,0.470787,0.229574 \backslash 0,0,0.920353,1.727038,-0.418342 \backslash \mathrm{H}, 0,1.437591$, $2.540646,-0.286281 \backslash \mathrm{H}, 0,0.512012,-0.672239,-1.187626 \backslash \mathrm{H}, 0,2.558594,-1.74$ $5991,-1.080379 \backslash \mathrm{H}, 0,2.683928,-1.605317,0.525425 \backslash \mathrm{H}, 0,0.138782,-1.776172$, $1.444876 \backslash \mathrm{H}, 0,-0.910882,1.059914,1.118186 \backslash \mathrm{H}, 0,-3.91394,0.409543,-0.5586$ $88 \backslash \mathrm{H}, 0,-1.703842,-0.258564,1.961471 \backslash \backslash$ Version=IA64L-G03RevC . 02 \State=2$\mathrm{A} \backslash \mathrm{HF}=-547.8770052 \backslash \mathrm{MP} 2=-549.3684367 \backslash \mathrm{RMSD}=4.163 e-09 \backslash \mathrm{PG}=\mathrm{C} 01 \quad[\mathrm{X}(\mathrm{C} 5 \mathrm{H} 8 \mathrm{~N} 104)]$ $\backslash \backslash @$

\section{Acrylic acid}

$1 \backslash 1 \backslash G I N C-L C 62 \backslash S P \backslash R O M P 2-F C \backslash 6-31 G(d) \backslash C 3 H 402 \backslash G M S 501 \backslash 23-F e b-2005 \backslash 0 \backslash \backslash \# R O M P 2$ /6-31G* SCF=TIGHT \\acrlyic acid Cs symm romp2/6-31G*//b3lyp/6-31G(d,p) $\backslash \backslash 0,1 \backslash \mathrm{C}, 0,0.419826,-1.924138,0 . \backslash \mathrm{H}, 0,0.104497,-2.9622,0 . \backslash \mathrm{H}, 0,1.485032,-$ $1.713793,0 . \backslash \mathrm{C}, 0,-0.461788,-0.922856,0 . \backslash \mathrm{H}, 0,-1.534159,-1.090009,0 . \backslash \mathrm{C}, 0$, $0 ., 0.48698,0 . \backslash 0,0,1.155088,0.860929,0 . \backslash 0,0,-1.04798,1.349702,0 . \backslash \mathrm{H}, 0,-0$ $.660462,2.241031,0 . \backslash \backslash$ Version=IA32L-G03RevC .02 $\backslash$ State $=1-A^{\prime} \backslash H F=-265.65143$ $78 \backslash M P 2=-266.3803828 \backslash \mathrm{RMSD}=7.451 \mathrm{e}-09 \backslash \mathrm{PG}=\mathrm{CS} \quad[\mathrm{SG}(\mathrm{C} 3 \mathrm{H} 4 \mathrm{O} 2)] \backslash \backslash @$ 


\section{TS: $2 a \rightarrow 3 a^{*}$}

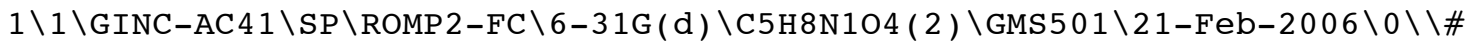

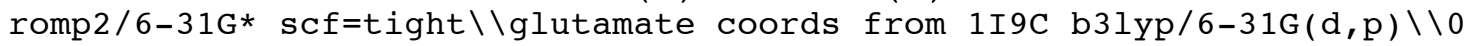
, $2 \backslash \mathrm{C}, 0,0.204431,0.626568,1.174272 \backslash \mathrm{C}, 0,-1.209317,0.65442,-0.460819 \backslash \mathrm{H}, 0$, $-0.428385,0.714475,-1.209151 \backslash \mathrm{N}, 0,-2.040621,1.741117,-0.338195 \backslash \mathrm{H}, 0,-2.8$ $09544,1.618795,0.309815 \backslash \mathrm{H}, 0,-1.627529,2.661617,-0.365326 \backslash \mathrm{C}, 0,-1.867621$ $,-0.632037,-0.20722 \backslash 0,0,-2.914606,-0.74621,0.413512 \backslash 0,0,-1.168654,-1.6$ $89304,-0.68147 \backslash \mathrm{H}, 0,-1.659677,-2.480646,-0.404486 \backslash \mathrm{C}, 0,1.219899,-0.27745$ $6,0.930116 \backslash \mathrm{C}, 0,2.285034,0.076724,0.001314 \backslash 0,0,2.33613,1.106071,-0.6607$ $17 \backslash 0,0,3.252065,-0.878142,-0.083289 \backslash \mathrm{H}, 0,0.402577,1.671475,0.95779 \backslash \mathrm{H}, 0$, $-0.520718,0.427055,1.957349 \backslash \mathrm{H}, 0,1.199978,-1.28808,1.321113 \backslash \mathrm{H}, 0,3.89360$ $9,-0.54115,-0.730003 \backslash \backslash$ Version=IA64L-G03RevD. $01 \backslash$ State $=2-A \backslash H F=-547.82953$ $92 \backslash \mathrm{MP} 2=-549.3479478 \backslash \mathrm{RMSD}=2.702 \mathrm{e}-09 \backslash \mathrm{Thermal}=0 . \backslash \mathrm{PG}=\mathrm{C} 01 \quad[\mathrm{X}(\mathrm{C} 5 \mathrm{H} 8 \mathrm{~N} 104)] \backslash \backslash @$

\section{TS: $3 a^{*} \rightarrow 4 a$}

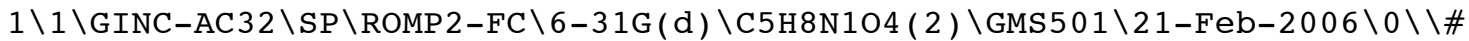
romp2/6-31G* scf=tight \radical product glutamate coords from $1 \mathrm{I} 9 \mathrm{C}$ TS

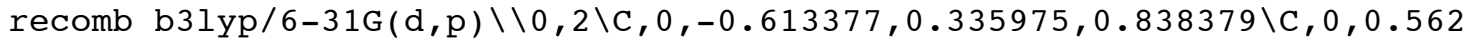
$656,-0.10861,-0.794523 \backslash \mathrm{H}, 0,0.165442,0.651103,-1.463115 \backslash \mathrm{N}, 0,0.402565,-1$ $.420548,-1.199827 \backslash \mathrm{H}, 0,-0.565439,-1.695669,-1.338797 \backslash \mathrm{H}, 0,0.90405,-2.066$ $299,-0.598052 \backslash 0,0,2.606257,-0.663527,0.298802 \backslash \mathrm{C}, 0,1.884157,0.180283,-0$ $.19907 \backslash 0,0,2.174965,1.504343,-0.192186 \backslash \mathrm{H}, 0,3.026338,1.590068,0.268398 \backslash$ C , $0,-1.87454,0.191006,0.072957 \backslash 0,0,-2.415609,-0.867559,-0.201325 \backslash 0,0,-$ $2.348984,1.379264,-0.377639 \backslash \mathrm{C}, 0,-0.38243,-0.587509,1.871814 \backslash \mathrm{H}, 0,-0.280$ $152,1.361735,0.975683 \backslash \mathrm{H}, 0,0.402336,-0.425117,2.600497 \backslash \mathrm{H}, 0,-0.917851,-1$ $.529541,1.892036 \backslash \mathrm{H}, 0,-3.144502,1.170528,-0.896423 \backslash \backslash$ Version=IA64L-G03Re $\mathrm{VD} .01 \backslash$ State $=2-\mathrm{A} \backslash \mathrm{HF}=-547.8288671 \backslash \mathrm{MP} 2=-549.3432414 \backslash \mathrm{RMSD}=8.006 \mathrm{e}-09 \backslash \mathrm{Therma}$ $1=0 . \backslash \mathrm{PG}=\mathrm{C} 01 \quad[\mathrm{X}(\mathrm{C} 5 \mathrm{H} 8 \mathrm{~N} 104)] \backslash \backslash @$

\section{4a}

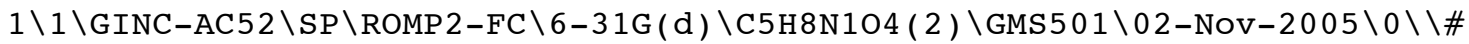
ROMP2/6-31G* SCF=TIGHT \radical product glutamate coords from 1 I9C b31 $\mathrm{yp} / 6-31 \mathrm{G}(\mathrm{d}, \mathrm{p}) \backslash \backslash 0,2 \backslash \mathrm{N}, 0,0.209647,0.30816,1.734875 \backslash \mathrm{C}, 0,-0.514031,0.14778$ $9,-0.623407 \backslash \mathrm{C}, 0,-1.940692,-0.132109,-0.160249 \backslash 0,0,-2.82053,0.686771,-0$ $.031386 \backslash 0,0,-2.133062,-1.453965,0.067033 \backslash \mathrm{C}, 0,0.484099,-0.35646,0.47545$ $9 \backslash \mathrm{C}, 0,-0.328641,1.584102,-0.975426 \backslash \mathrm{C}, 0,1.930644,-0.1996,-0.002731 \backslash 0,0$, $2.785193,0.441554,0.566406 \backslash 0,0,2.167736,-0.886908,-1.146757 \backslash \mathrm{H}, 0,3.1045$ $52,-0.743632,-1.365375 \backslash \mathrm{H}, 0,0.308605,-1.431305,0.597482 \backslash \mathrm{H}, 0,-0.333547,-$ $0.488702,-1.502839 \backslash \mathrm{H}, 0,0.858737,-0.023145,2.444142 \backslash \mathrm{H}, 0,0.395509,1.3043$ $, 1.632365 \backslash \mathrm{H}, 0,0.590979,1.910947,-1.447072 \backslash \mathrm{H}, 0,-1.124718,2.29575,-0.801$ $769 \backslash \mathrm{H}, 0,-3.050605,-1.543275,0.374698 \backslash \backslash$ Version=IA64L-G03RevC .02 $\backslash$ State $=2$ $-\mathrm{A} \backslash \mathrm{HF}=-547.8747605 \backslash \mathrm{MP} 2=-549.3687042 \backslash \mathrm{RMSD}=7.441 \mathrm{e}-09 \backslash \mathrm{PG}=\mathrm{C} 01 \quad[\mathrm{X}(\mathrm{C} 5 \mathrm{H} 8 \mathrm{~N} 104)$ ]$\backslash \backslash Q$

\section{$5 a$}

$1 \backslash 1 \backslash G I N C-A C 50 \backslash S P \backslash R O M P 2-F C \backslash 6-31 G(d) \backslash C 5 H 9 N 104 \backslash G M S 501 \backslash 02-N o v-2005 \backslash 0 \backslash \backslash \# R O M$ P2/6-31G* SCF=TIGHT \product glutamate coords from 1I9C b3lyp/6-31G(d, p) $\backslash \backslash 0,1 \backslash \mathrm{N}, 0,0.277691,-0.13686,1.781292 \backslash \mathrm{C}, 0,-0.511227,0.287347,-0.53055$ $7 \backslash \mathrm{C}, 0,-1.935159,-0.132133,-0.18916 \backslash 0,0,-2.862384,0.617529,0.013876 \backslash 0,0$ $,-2.079691,-1.481824,-0.183401 \backslash \mathrm{C}, 0,0.49384,-0.474405,0.382123 \backslash \mathrm{C}, 0,-0.3$ $68778,1.809777,-0.456212 \backslash \mathrm{C}, 0,1.927135,-0.207487,-0.083204 \backslash 0,0,2.763729$ $, 0.394757,0.553276 \backslash 0,0,2.167764,-0.715716,-1.315192 \backslash \mathrm{H}, 0,3.087349,-0.48$ $6547,-1.534052 \backslash \mathrm{H}, 0,0.30422,-1.542902,0.245167 \backslash \mathrm{H}, 0,-0.329428,-0.051752$, $-1.558643 \backslash \mathrm{H}, 0,0.708017,-0.843025,2.37236 \backslash \mathrm{H}, 0,0.768857,0.728934,1.99449$ $4 \backslash \mathrm{H}, 0,0.636873,2.124125,-0.750548 \backslash \mathrm{H}, 0,-0.568703,2.168669,0.556416 \backslash \mathrm{H}, 0$, $-3.011203,-1.648392,0.037822 \backslash \mathrm{H}, 0,-1.090021,2.292364,-1.118464 \backslash \backslash$ Version $=\mathrm{IA} 64 \mathrm{~L}-\mathrm{G} 03 \mathrm{RevC} .02 \backslash \mathrm{State}=1-\mathrm{A} \backslash \mathrm{HF}=-548.5115285 \backslash \mathrm{MP} 2=-550.0290212 \backslash \mathrm{RMSD}=4.24$ $8 e-09 \backslash P G=C 01 \quad[X(C 5 H 9 N 104)] \backslash \backslash @$ 


\section{1b}

$1 \backslash 1 \backslash G I N C-B A R O S S A 106 \backslash S P \backslash R O M P 2-F C \backslash 6-31 G(d) \backslash C 5 H 805 \backslash S A N D A L G \backslash 30-J u n-2005 \backslash 0 \backslash$

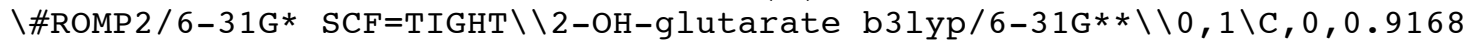
$4,-0.016606,0.477438 \backslash \mathrm{C}, 0,2.380429,-0.194998,0.020482 \backslash 0,0,2.925219,-1.2$ $74219,-0.005163 \backslash 0,0,2.977247,0.945568,-0.345026 \backslash \mathrm{H}, 0,2.294222,1.641766$, $-0.230864 \backslash \mathrm{C}, 0,0.011539,-0.911655,-0.383188 \backslash 0,0,0.611491,1.370803,0.436$ $043 \backslash \mathrm{H}, 0,0.879188,-0.37572,1.518111 \backslash \mathrm{H}, 0,0.503159,-1.887133,-0.4276 \backslash \mathrm{H}, 0$, $-0.012802,-0.519111,-1.406834 \backslash \mathrm{C}, 0,-1.418947,-1.118823,0.134844 \backslash \mathrm{C}, 0,-2$. $349553,0.053698,-0.069545 \backslash \mathrm{H}, 0,-1.416688,-1.345779,1.209903 \backslash \mathrm{H}, 0,-1.8799$ $08,-1.987611,-0.346221 \backslash 0,0,-2.016369,1.203918,-0.307678 \backslash 0,0,-3.640833$, $-0.296281,0.044844 \backslash \mathrm{H}, 0,-4.166701,0.513805,-0.073572 \backslash \mathrm{H}, 0,-0.296359,1.49$ $1771,0.092739 \backslash \backslash$ Version=IA32L-G03RevC.02 $\backslash$ State $=1-A \backslash H F=-568.3406207 \backslash M P 2=$ $-569.8716786 \backslash \mathrm{RMSD}=4.304 \mathrm{e}-09 \backslash \mathrm{PG}=\mathrm{C} 01 \quad[\mathrm{X}(\mathrm{C} 5 \mathrm{H} 8 \mathrm{O} 5)] \backslash \backslash \mathrm{a}$

\section{2b}

$1 \backslash 1 \backslash G I N C-B A R O S S A 021 \backslash S P \backslash R O M P 2-F C \backslash 6-31 G(d) \backslash C 5 H 7 O 5$ ( 2 ) \SANDALG $\backslash 30-J u n-2005$

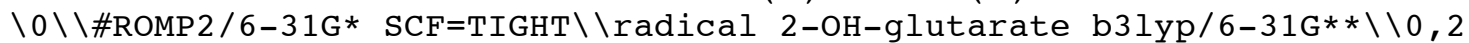
$\backslash \mathrm{C}, 0,0.880096,-0.030868,0.48114 \backslash \mathrm{C}, 0,2.355359,-0.194321,0.053785 \backslash 0,0,2$. $908774,-1.268353,0.036083 \backslash \mathrm{O}, 0,2.945639,0.953327,-0.297493 \backslash \mathrm{H}, 0,2.256302$ $, 1.644666,-0.195636 \backslash \mathrm{C}, 0,0.001851,-0.92313,-0.442607 \backslash 0,0,0.553013,1.345$ $676,0.437055 \backslash \mathrm{H}, 0,0.808856,-0.409317,1.510742 \backslash \mathrm{H}, 0,0.496281,-1.894867,-0$ $.501637 \backslash \mathrm{H}, 0,0.020575,-0.466961,-1.441778 \backslash \mathrm{C}, 0,-1.405491,-1.088219,0.014$ $783 \backslash \mathrm{C}, 0,-2.353411,-0.000745,-0.084118 \backslash \mathrm{H}, 0,-1.749611,-2.024642,0.441004$ $\backslash 0,0,-2.057579,1.157508,-0.394881 \backslash 0,0,-3.623439,-0.355073,0.201109 \backslash \mathrm{H}, 0$ $,-4.162916,0.449284,0.119154 \backslash \mathrm{H}, 0,-0.35117,1.460865,0.075267 \backslash \backslash$ Version=I A32L-G03RevC.02 State $=2-A \backslash H F=-567.71377 \backslash M P 2=-569.2246378 \backslash \mathrm{RMSD}=9.024 \mathrm{e}-0$ $9 \backslash \mathrm{PG}=\mathrm{C} 01 \quad[\mathrm{X}(\mathrm{C} 5 \mathrm{H} 705)] \backslash \backslash @$

\section{C3-radical derived from $\mathbf{1 b}$}

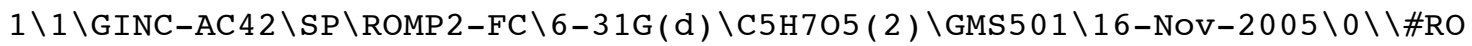

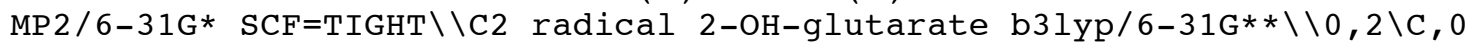
$, 0.964037,-0.654996,-0.171583 \backslash \mathrm{C}, 0,1.905821,0.554759,-0.151541 \backslash 0,0,3.09$ $8116,0.4663,0.057444 \backslash \mathrm{C}, 0,-0.012149,-0.576553,0.968079 \backslash \mathrm{C}, 0,-1.232718,0$. $283363,0.962195 \backslash \mathrm{C}, 0,-2.234137,-0.029841,-0.144758 \backslash 0,0,-1.990741,-0.547$ $645,-1.211848 \backslash 0,0,-3.47542,0.393526,0.192671 \backslash 0,0,1.276492,1.725773,-0$. $371298 \backslash \mathrm{H}, 0,1.953606,2.422232,-0.318841 \backslash \mathrm{H}, 0,0.40846,-0.62388,-1.121116 \backslash$ $\mathrm{H}, 0,0.247611,-1.142436,1.856194 \backslash \mathrm{H}, 0,-0.970167,1.344723,0.813356 \backslash \mathrm{H}, 0,-4$ $.044793,0.198758,-0.571578 \backslash \mathrm{H}, 0,-1.754436,0.231662,1.920889 \backslash 0,0,1.73699$ $9,-1.831445,-0.094664 \backslash \mathrm{H}, 0,2.651032,-1.543534,0.068301 \backslash \backslash$ Version=IA $64 \mathrm{~L}-\mathrm{G}$ 03 RevC . 02 \State $=2-A \backslash H F=-567.7049134 \backslash M P 2=-569.2099635 \backslash R M S D=4.866 e-09 \backslash P G$ $=\mathrm{C} 01[\mathrm{X}(\mathrm{C} 5 \mathrm{H} 705)] \backslash \backslash \mathrm{Q}$

\section{$T S: 2 b \rightarrow 3 b^{*}$}

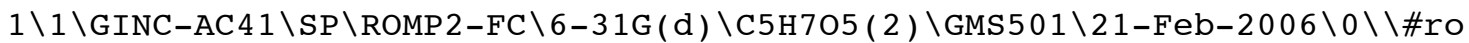
$\mathrm{mp} 2 / 6-31 \mathrm{G} * \mathrm{scf}=\mathrm{tight} \backslash \backslash \mathrm{radical} 2-\mathrm{OH}-\mathrm{glutarate} \mathrm{b} 31 \mathrm{yp} / 6-31 \mathrm{G} * * \backslash \backslash 0,2 \backslash \mathrm{C}, 0,-0$ $.234738,-0.814463,1.137035 \backslash \mathrm{C}, 0,1.239893,-0.653384,-0.492217 \backslash \mathrm{H}, 0,0.4842$ $06,-0.79401,-1.254814 \backslash 0,0,2.077602,-1.692332,-0.307244 \backslash \mathrm{H}, 0,2.842813,-1$ $.328504,0.182728 \backslash 0,0,2.899328,0.756867,0.383503 \backslash \mathrm{C}, 0,1.827089,0.653543$, $-0.204227 \backslash \mathrm{O}, 0,1.0751,1.703497,-0.586857 \backslash \mathrm{H}, 0,1.545437,2.501821,-0.29413$ $8 \backslash \mathrm{C}, 0,-1.226702,0.122938,0.980238 \backslash \mathrm{C}, 0,-2.273095,-0.087427,-0.020614 \backslash 0$, $0,-2.304908,-1.004873,-0.827048 \backslash 0,0,-3.232072,0.875124,0.018135 \backslash \mathrm{H}, 0,-0$ $.397889,-1.81712,0.756913 \backslash \mathrm{H}, 0,0.489526,-0.711401,1.938884 \backslash \mathrm{H}, 0,-1.21624$ $9,1.068942,1.509756 \backslash \mathrm{H}, 0,-3.862924,0.646774,-0.68453 \backslash \backslash$ Version=IA64L-G03 RevD. $01 \backslash$ State $=2-A \backslash H F=-567.6561304 \backslash M P 2=-569.1834539 \backslash$ RMSD $=5.516 \mathrm{e}-09 \backslash \mathrm{Ther}$ $\mathrm{mal}=0 . \backslash \mathrm{PG}=\mathrm{C} 01 \quad \mathrm{X}(\mathrm{C} 5 \mathrm{H} 705)] \backslash \backslash @$ 


\section{$T S: 3 b^{*} \rightarrow 4 b$}

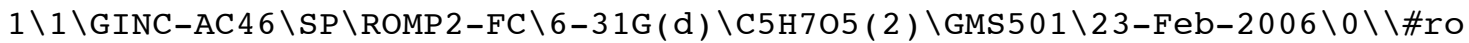
mp2 /6-31G* scf=(tight, noincfock, novaracc, maxcycle $=5000$, vshift $=1500$, nod iis) guess=read geom=check $\backslash \backslash$ radical from product 2-OH-glutarate 21 b31

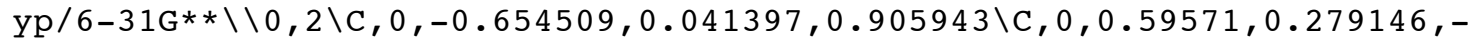
$0.78927 \backslash 0,0,0.360765,-0.721841,-1.66553 \backslash \mathrm{H}, 0,0.97598,-1.440916,-1.42652$ $4 \backslash \mathrm{H}, 0,0.206068,1.242321,-1.10015 \backslash \mathrm{C}, 0,1.901068,0.20182,-0.119708 \backslash 0,0,2$. $245847,1.322769,0.549368 \backslash \mathrm{H}, 0,3.08687,1.129656,0.996483 \backslash 0,0,2.562464,-0$ $.825852,-0.112613 \backslash \mathrm{C}, 0,-1.932272,0.128609,0.140025 \backslash 0,0,-2.632543,-0.805$ $873,-0.182668 \backslash 0,0,-2.231534,1.414228,-0.189506 \backslash \mathrm{C}, 0,-0.382588,-1.159033$ $, 1.55243 \backslash \mathrm{H}, 0,-0.308915,0.97435,1.343935 \backslash \mathrm{H}, 0,0.420161,-1.24034,2.275825$ $\backslash \mathrm{H}, 0,-0.924279,-2.056494,1.276976 \backslash \mathrm{H}, 0,-3.060329,1.372343,-0.69546 \backslash \backslash$ Ver sion=IA6 $4 \mathrm{~L}-\mathrm{G} 03$ RevD $.01 \backslash$ State $=2-\mathrm{A} \backslash \mathrm{HF}=-567.6438831 \backslash \mathrm{MP} 2=-569.1760542 \backslash \mathrm{RMSD}=$ $9.634 \mathrm{e}-09 \backslash \mathrm{Thermal}=0 . \backslash \mathrm{PG}=\mathrm{C} 01 \quad[\mathrm{X}(\mathrm{C} 5 \mathrm{H} 7 \mathrm{O} 5)] \backslash \backslash @$

\section{4b}

$1 \backslash 1 \backslash G I N C-B A R O S S A 050 \backslash S P \backslash R O M P 2-F C \backslash 6-31 G$ (d) \C5H7O5 ( 2) \SANDALG $30-J u n-2005$

$\backslash 0 \backslash \backslash \# R O M P 2 / 6-31 G *$ SCF=TIGHT \\radical from product 2-OH-glutarate 21 b3 $1 \mathrm{yp} / 6-31 \mathrm{G} * * \backslash \backslash 0,2 \backslash \mathrm{H}, 0,0.093912,-0.03864,2.195561 \backslash \mathrm{C}, 0,0.424531,-0.840108$ $, 1.545971 \backslash \mathrm{C}, 0,0.540989,-0.594164,0.072604 \backslash \mathrm{H}, 0,0.792702,-1.760803,1.979$ $975 \backslash \mathrm{C}, 0,1.906299,0.038308,-0.196608 \backslash \mathrm{C}, 0,-0.558752,0.35066,-0.464887 \backslash \mathrm{H}$, $0,0.483851,-1.540609,-0.473672 \backslash 0,0,2.108959,1.23712,-0.299399 \backslash 0,0,2.89$ $2511,-0.870001,-0.27021 \backslash \mathrm{H}, 0,3.723667,-0.377512,-0.388287 \backslash 0,0,-0.579794$ $, 1.596592,0.211027 \backslash \mathrm{C}, 0,-1.938721,-0.304889,-0.312724 \backslash \mathrm{H}, 0,-0.397278,0.4$ $94541,-1.54386 \backslash 0,0,-2.17296,-1.411021,-0.736391 \backslash 0,0,-2.853318,0.453682$ , $0.308293 \backslash \mathrm{H}, 0,-2.412929,1.288883,0.558091 \backslash \mathrm{H}, 0,0.306825,1.984327,0.0994$

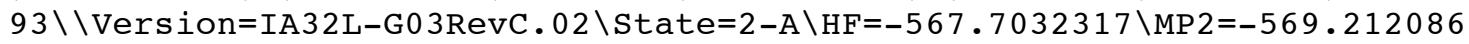
$1 \backslash \mathrm{RMSD}=4.072 \mathrm{e}-09 \backslash \mathrm{PG}=\mathrm{C} 01[\mathrm{X}(\mathrm{C} 5 \mathrm{H} 705)] \backslash \backslash @$

\section{$5 b$}

$1 \backslash 1 \backslash G I N C-B A R O S S A 088 \backslash S P \backslash R O M P 2-F C \backslash 6-31 G(d) \backslash C 5 H 805 \backslash S A N D A L G \backslash 30-J u n-2005 \backslash 0 \backslash$

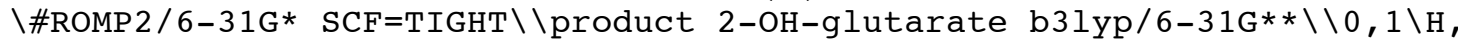
$0,0.399897,-0.237995,2.119603 \backslash \mathrm{C}, 0,0.419987,-1.079574,1.422608 \backslash \mathrm{H}, 0,1.25$ $3633,-1.735811,1.682706 \backslash \mathrm{C}, 0,0.55266,-0.579121,-0.030867 \backslash \mathrm{H}, 0,-0.507311$, $-1.6473,1.530965 \backslash \mathrm{C}, 0,1.898748,0.091652,-0.223391 \backslash \mathrm{C}, 0,-0.560115,0.41178$ $3,-0.428919 \backslash \mathrm{H}, 0,0.494272,-1.438289,-0.705923 \backslash 0,0,2.085687,1.297933,-0$. $247804 \backslash 0,0,2.908049,-0.788722,-0.339925 \backslash \mathrm{H}, 0,3.727584,-0.269842,-0.4181$ $63 \backslash 0,0,-0.581471,1.571831,0.389733 \backslash \mathrm{C}, 0,-1.941263,-0.25185,-0.345679 \backslash \mathrm{H}$, $0,-0.416738,0.689358,-1.484812 \backslash 0,0,-2.163727,-1.342047,-0.816925 \backslash 0,0,-$ $2.876305,0.484421,0.271164 \backslash \mathrm{H}, 0,-2.437402,1.299669,0.583731 \backslash \mathrm{H}, 0,0.28810$ $2,1.995546,0.279443 \backslash \backslash$ Version=IA32L-G03RevC . 02 \State=1-A \HF=-568.340916 $6 \backslash M P 2=-569.873538 \backslash \mathrm{RMSD}=4.647 \mathrm{e}-09 \backslash \mathrm{PG}=\mathrm{C} 01 \quad[\mathrm{X}(\mathrm{C} 5 \mathrm{H} 805)] \backslash \backslash @$

\section{$\mathrm{H}-\mathrm{HC}\left(\mathrm{CO}_{2} \mathrm{H}\right)-\mathrm{H}$}

$1 \backslash 1 \backslash G I N C-S C 107 \backslash S P \backslash R O M P 2-F C \backslash 6-31 G(d) \backslash C 2 H 402 \backslash G M S 501 \backslash 16-M a r-2005 \backslash 0 \backslash \backslash \#$ ROM

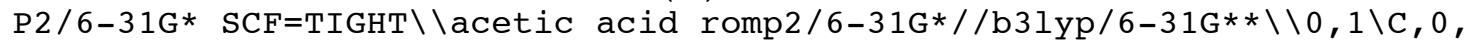
0 . $0.155438,0 . \backslash 0,0,0.194419,1.34993,0 . \backslash 0,0,-1.247806,-0.380001,0 . \backslash \mathrm{H}, 0$, $-1.861038,0.374334,0 . \backslash \mathrm{C}, 0,1.059182,-0.917045,0 . \backslash \mathrm{H}, 0,2.044919,-0.454209$ , $0 . \backslash \mathrm{H}, 0,0.94406,-1.554957,0.88114 \backslash \mathrm{H}, 0,0.94406,-1.554957,-0.88114 \backslash \backslash$ Vers ion=Al 64 T-G03RevC.02\State $=1-\mathrm{A}^{\prime} \backslash \mathrm{HF}=-227.8081958 \backslash \mathrm{MP} 2=-228.418731 \backslash \mathrm{RMSD}=2$ $.623 e-09 \backslash \mathrm{PG}=\mathrm{CS}[\mathrm{SG}(\mathrm{C} 2 \mathrm{H} 2 \mathrm{O} 2), \mathrm{X}(\mathrm{H} 2)] \backslash \backslash @$

\section{$\mathrm{H}-\cdot \mathrm{C}\left(\mathrm{CO}_{2} \mathrm{H}\right)-\mathrm{H}$}

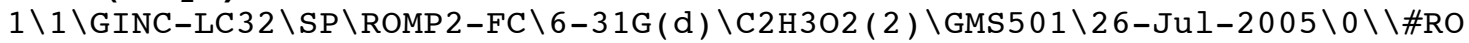
MP2 /6-31G* SCF $=$ TIGHT MAXDISK=39321600\\acetic acid radical Cs symm b31

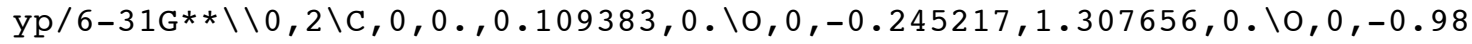
$073,-0.836454,0 . \backslash \mathrm{H}, 0,-1.819634,-0.346964,0 . \backslash \mathrm{C}, 0,1.328875,-0.460244,0 . \backslash$ $\mathrm{H}, 0,2.173961,0.214941,0 . \backslash \mathrm{H}, 0,1.480005,-1.532427,0 . \backslash \backslash$ Version=IA32L-G03R evC.02 $\backslash$ State $=2-A " \backslash H F=-227.1758341 \backslash M P 2=-227.7644211 \backslash$ RMSD=3.815e-09 $\backslash P G=C$ $S[S G(C 2 \mathrm{H} 3 \mathrm{O} 2)] \backslash \backslash @$ 


\section{$\mathrm{H}-\mathrm{HC}\left(\mathrm{CO}_{2} \mathrm{H}\right)-\mathrm{NH}_{2}$}

$1 \backslash 1 \backslash G I N C-L C 64 \backslash S P \backslash R O M P 2-F C \backslash 6-31 G(d) \backslash C 2 H 5 N 102 \backslash G M S 501 \backslash 18-F e b-2005 \backslash 0 \backslash \backslash \# R O M$

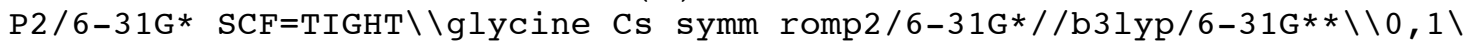
$\mathrm{C}, 0,-0.580548,-0.858177,0 . \backslash \mathrm{C}, 0,0 ., 0.551593,0 . \backslash 0,0,-0.972112,1.494684,0$ $. \backslash 0,0,1.180876,0.820624,0 . \backslash \mathrm{H}, 0,-0.519556,2.355343,0 . \backslash \mathrm{N}, 0,0.398539,-1.9$ $28701,0 . \backslash \mathrm{H}, 0,1.009427,-1.819593,0.806671 \backslash \mathrm{H}, 0,1.009427,-1.819593,-0.806$ $671 \backslash \mathrm{H}, 0,-1.237946,-0.9491,0.873037 \backslash \mathrm{H}, 0,-1.237946,-0.9491,-0.873037 \backslash \backslash \mathrm{Ve}$ rsion=IA32L-G03RevC.02 \State $=1-A^{\prime} \backslash \mathrm{HF}=-282.8280465 \backslash M P 2=-283.6003968 \backslash \mathrm{RMS}$ $\mathrm{D}=6.032 \mathrm{e}-09 \backslash \mathrm{PG}=\mathrm{CS}[\mathrm{SG}(\mathrm{C} 2 \mathrm{H} 1 \mathrm{~N} 102), \mathrm{X}(\mathrm{H} 4)] \backslash \backslash @$

\section{$\mathrm{H}-\cdot \mathrm{C}\left(\mathrm{CO}_{2} \mathrm{H}\right)-\mathrm{NH}_{2}(3 \mathrm{a})$}

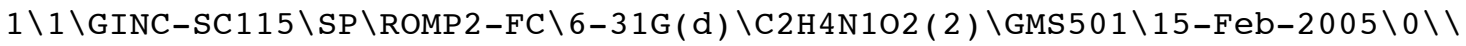
\#ROMP2/6-31G* SCF=TIGHT \glycyl radical C1 symm romp2/6-31G*//b3lyp/6$31 \mathrm{G} * * \backslash \backslash 0,2 \backslash \mathrm{C}, 0,-0.71432,-0.681982,0.010147 \backslash \mathrm{H}, 0,-0.734036,-1.762793,0.0$ $11499 \backslash \mathrm{N}, 0,-1.885231,0.012381,-0.059922 \backslash \mathrm{H}, 0,-2.748024,-0.419205,0.23086$ $2 \backslash \mathrm{H}, 0,-1.798882,1.014253,0.061776 \backslash \mathrm{C}, 0,0.50125,0.073143,0.002227 \backslash 0,0,0$. $548358,1.304434,0.007943 \backslash \mathrm{O}, 0,1.625398,-0.703163,-0.003095 \backslash \mathrm{H}, 0,2.36593$, $-0.076055,0.002285 \backslash \backslash$ Version=Al64T-G03RevC.02 $\backslash$ State $=2-\mathrm{A} \backslash \mathrm{HF}=-282.2134367$ $\backslash \mathrm{MP} 2=-282.9752382 \backslash \mathrm{RMSD}=7.657 \mathrm{e}-09 \backslash \mathrm{PG}=\mathrm{C} 01 \quad[\mathrm{X}(\mathrm{C} 2 \mathrm{H} 4 \mathrm{~N} 102)] \backslash \backslash @$

\section{$\mathrm{H}-\mathrm{HC}\left(\mathrm{CO}_{2} \mathrm{H}\right)-\mathrm{SH}$}

$1 \backslash 1 \backslash G I N C-A C 14 \backslash S P \backslash R O M P 2-F C \backslash 6-31 G(d) \backslash C 2 H 4 O 2 S 1 \backslash G M S 501 \backslash 21-$ Sep-2005 0 ( 1 \#ROM

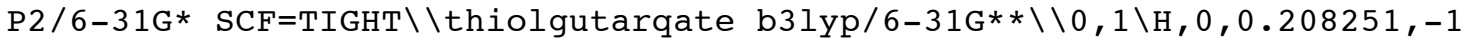
$.159987,1.272024 \backslash \mathrm{H}, 0,0.152291,-1.6743,-0.411879 \backslash \mathrm{C}, 0,0.241384,-0.801483$ $, 0.237207 \backslash \mathrm{C}, 0,-0.955893,0.108395,0.032523 \backslash 0,0,-0.947605,1.317046,0.075$ $029 \backslash 0,0,-2.080249,-0.617435,-0.156767 \backslash \mathrm{H}, 0,-2.810022,0.021187,-0.232706$ $\backslash \mathrm{S}, 0,1.844482,0.009038,-0.117917 \backslash \mathrm{H}, 0,1.447649,1.230125,0.294755 \backslash \backslash V e r s i$

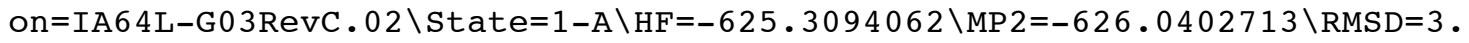
$578 e-09 \backslash P G=C 01 \quad[X(C 2 H 402 S 1)] \backslash \backslash @$

\section{$\mathrm{H}-\cdot \mathrm{C}\left(\mathrm{CO}_{2} \mathrm{H}\right)-\mathrm{SH}(3 \mathrm{C})$}

$1 \backslash 1 \backslash G I N C-A C 9 \backslash S P \backslash R O M P 2-F C \backslash 6-31 G(d) \backslash C 2 H 302 S 1$ ( 2) \GMS501\20-Sep-2005 $10 \backslash \backslash \# R$

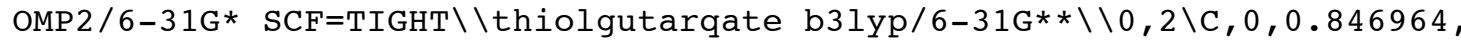
$-0.377501,0 . \backslash 0,0,0.44712,-1.536655,0 . \backslash 0,0,2.168867,-0.060081,0 . \backslash \mathrm{H}, 0,2$. $643159,-0.907598,0 . \backslash \mathrm{C}, 0,0.0 .793991,0 . \backslash \mathrm{S}, 0,-1.712372,0.632104,0 . \backslash \mathrm{H}, 0,-$ $1.672884,-0.72284,0 . \backslash \mathrm{H}, 0,0.417992,1.791722,0 . \backslash \backslash$ Version=IA64L-G03RevC.0 $2 \backslash$ State $=2-\mathrm{A} " \backslash \mathrm{HF}=-624.6900459 \backslash \mathrm{MP} 2=-625.4084624 \backslash \mathrm{RMSD}=5.210 \mathrm{e}-09 \backslash \mathrm{PG}=\mathrm{CS}$ [SG ( C2H3O2S1) ] \\@

\section{$\mathrm{H}-\mathrm{HC}\left(\mathrm{CO}_{2} \mathrm{H}\right)-\mathrm{S}^{-}$}

$1 \backslash 1 \backslash G I N C-A C 52 \backslash S P \backslash R O M P 2-F C \backslash 6-31 G(d) \backslash C 2 H 3 O 2 S 1(1-) \backslash G M S 501 \backslash 16-M a y-2006 \backslash 0 \backslash \backslash$

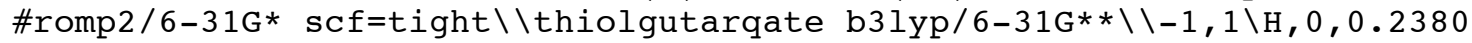
$29,1.544579,0.875191 \backslash \mathrm{H}, 0,0.238029,1.544579,-0.875191 \backslash \mathrm{C}, 0,0 ., 0.924926,0$ $. \backslash \mathrm{C}, 0,1.029087,-0.219264,0 . \backslash 0,0,0.495038,-1.440351,0 . \backslash 0,0,2.237089,-0$. $032103,0 . \backslash \mathrm{S}, 0,-1.749938,0.356398,0 . \backslash \mathrm{H}, 0,-0.508583,-1.245871,0$. \\Versio $\mathrm{n}=\mathrm{IA} 64 \mathrm{~L}-\mathrm{G} 03 \mathrm{RevD} .01 \backslash$ State $=1-\mathrm{A}^{\prime} \backslash \mathrm{HF}=-624.7591961 \backslash \mathrm{MP} 2=-625.4947398 \backslash \mathrm{RMSD}=3$. $352 \mathrm{e}-09 \backslash \mathrm{Thermal}=0 . \backslash \mathrm{PG}=\mathrm{CS} \quad[\mathrm{SG}(\mathrm{C} 2 \mathrm{H} 102 \mathrm{~S} 1), \mathrm{X}(\mathrm{H} 2)] \backslash \backslash @$

\section{$\mathrm{H}-\cdot \mathrm{C}\left(\mathrm{CO}_{2} \mathrm{H}\right)-\mathrm{S}^{-}(3 \mathrm{~d})$}

$1 \backslash 1 \backslash G I N C-A C 39 \backslash S P \backslash R O M P 2-F C \backslash 6-31 G(d) \backslash C 2 H 2 O 2 S 1(1-, 2) \backslash G M S 501 \backslash 16-M a y-2006 \backslash 0$

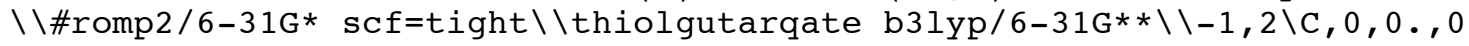
$.817697,0 . \backslash \mathrm{C}, 0,0.980878,-0.241757,0 . \backslash 0,0,0.471123,-1.518773,0 . \backslash 0,0,2.2$ $05683,-0.080915,0 . \backslash \mathrm{S}, 0,-1.700577,0.556277,0 . \backslash \mathrm{H}, 0,-0.504413,-1.382377,0$ . $\backslash \mathrm{H}, 0,0.413929,1.823811,0 . \backslash \backslash$ Version=IA64L-G03RevD . $01 \backslash$ State=2-A" $\backslash H F=-62$ $4.1565698 \backslash \mathrm{MP} 2=-624.8832045 \backslash \mathrm{RMSD}=2.471 \mathrm{e}-09 \backslash \mathrm{Thermal}=0 . \backslash \mathrm{PG}=\mathrm{CS} \quad[\mathrm{SG}(\mathrm{C} 2 \mathrm{H} 2 \mathrm{O} 2 \mathrm{~S}$ $1)] \backslash \backslash @$ 


\section{$\mathrm{H}-\mathrm{HC}\left(\mathrm{CO}_{2} \mathrm{H}\right)-\mathrm{OH}$}

$1 \backslash 1 \backslash G I N C-L C 123 \backslash S P \backslash R O M P 2-F C \backslash 6-31 G(d) \backslash C 2 H 4 O 3 \backslash G M S 501 \backslash 29-J u n-2005 \backslash 0 \backslash \backslash \#$ ROM P2/6-31G* SCF=TIGHT MAXDISK=65536000 \lcleavage product b3lyp/6-31G** 2 -hydroxyglutarate $\backslash \backslash 0,1 \backslash \mathrm{H}, 0,-1.255717,-0.955056,0.882628 \backslash \mathrm{H}, 0,-1.255717$, $-0.955056,-0.882628 \backslash \mathrm{C}, 0,-0.604327,-0.867126,0 . \backslash \mathrm{C}, 0,0 ., 0.522897,0 . \backslash 0,0$, $1.196821,0.72464,0 . \backslash 0,0,-0.925548,1.498658,0 . \backslash \mathrm{H}, 0,-0.44473,2.344012,0$. $\backslash 0,0,0.396329,-1.848326,0 . \backslash \mathrm{H}, 0,1.241314,-1.368303,0 . \backslash \backslash$ Version=IA32L-G0 3 RevC. 02 \State $=1-A^{\prime} \backslash \mathrm{HF}=-302.6535406 \backslash M P 2=-303.4400314 \backslash \mathrm{RMSD}=2.879 \mathrm{e}-09 \backslash \mathrm{PG}$ $=\mathrm{CS}[\mathrm{SG}(\mathrm{C} 2 \mathrm{H} 2 \mathrm{O} 3), \mathrm{X}(\mathrm{H} 2)] \backslash \backslash @$

\section{$\mathrm{H}-\cdot \mathrm{C}\left(\mathrm{CO}_{2} \mathrm{H}\right)-\mathrm{OH}(3 \mathrm{~b})$}

$1 \backslash 1 \backslash G I N C-B A R O S S A 125 \backslash S P \backslash R O M P 2-F C \backslash 6-31 G(d) \backslash C 2 H 3 O 3$ ( 2$) \backslash S A N D A L G \backslash 28-J u n-2005$ $\backslash 0 \backslash \backslash \#$ ROMP2/6-31G* SCF=TIGHT \\cleavage product b3lyp/6-31G** 2-hydroxy glutarate $\backslash \backslash 0,2 \backslash C, 0,0 ., 0.482886,0 . \backslash 0,0,1.226805,0.615074,0 . \backslash 0,0,-0.8674$ $21,1.52345,0 . \backslash \mathrm{H}, 0,-0.322625,2.3271,0 . \backslash \mathrm{C}, 0,-0.611859,-0.813489,0 . \backslash 0,0,0$ $.209755,-1.868262,0 . \backslash \mathrm{H}, 0,1.11327,-1.486427,0 . \backslash \mathrm{H}, 0,-1.6726,-1.019147,0$. $\backslash \backslash$ Version=IA32L-G03RevC.02 $\backslash$ State $=2-\mathrm{A} " \backslash \mathrm{HF}=-302.0362435 \backslash \mathrm{MP} 2=-302.8112572$ $\backslash \mathrm{RMSD}=2.299 \mathrm{e}-09 \backslash \mathrm{PG}=\mathrm{CS}[\mathrm{SG}(\mathrm{C} 2 \mathrm{H} 3 \mathrm{O} 3)] \backslash \backslash @$

\section{$\mathrm{H}-\mathrm{HC}\left(\mathrm{CO}_{2} \mathrm{H}\right)-\mathrm{O}^{-}$}

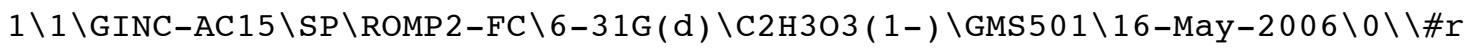
omp2/6-31G* scf=tight \cleavage product b3lyp/6-31G** 2-hydroxyglutara te $\backslash \backslash-1,1 \backslash \mathrm{H}, 0,-1.395673,-0.785409,0.884574 \backslash \mathrm{H}, 0,-1.395673,-0.785409,-0.8$ $84574 \backslash \mathrm{C}, 0,-0.736388,-0.735882,0 . \backslash \mathrm{C}, 0,0 ., 0.647141,0 . \backslash 0,0,1.26443,0.5248$ $15,0 . \backslash 0,0,-0.705767,1.675178,0 . \backslash \mathrm{O}, 0,0.213645,-1.784838,0 . \backslash \mathrm{H}, 0,1.031207$ $,-1.217975,0 . \backslash \backslash$ Version=IA64L-G03RevD.01 \State=1-A $\backslash \mathrm{HF}=-302.0898106 \backslash \mathrm{MP} 2$ $=-302.8873519 \backslash \mathrm{RMSD}=4.827 \mathrm{e}-09 \backslash \mathrm{Thermal}=0 . \backslash \mathrm{PG}=\mathrm{CS} \quad[\mathrm{SG}(\mathrm{C} 2 \mathrm{H} 1 \mathrm{O} 3), \mathrm{X}(\mathrm{H} 2)] \backslash \backslash @$

\section{$\mathrm{H}-\cdot \mathrm{C}\left(\mathrm{CO}_{2} \mathrm{H}\right)-\mathrm{O}^{-}$}

$1 \backslash 1 \backslash \mathrm{GINC}-\mathrm{AC} 2 \backslash \mathrm{SP} \backslash \mathrm{ROMP} 2-\mathrm{FC} \backslash 6-31 \mathrm{G}(\mathrm{d}) \backslash \mathrm{C} 2 \mathrm{H} 2 \mathrm{O} 3(1-, 2) \backslash \mathrm{GMS} 501 \backslash 16-M a y-2006 \backslash 0 \backslash \backslash \#$ romp2/6-31G* scf=tight \cleavage product b3lyp/6-31G** 2-hydroxygluta rate $\backslash \backslash-1,2 \backslash C, 0,0.0 .566305,0 . \backslash 0,0,1.349738,0.223613,0 . \backslash 0,0,-0.350572,1$ $.757468,0 . \backslash \mathrm{C}, 0,-0.824729,-0.612994,0 . \backslash \mathrm{O}, 0,-0.299417,-1.793863,0 . \backslash \mathrm{H}, 0,-$ $1.917316,-0.456667,0 . \backslash \mathrm{H}, 0,1.267704,-0.760943,0 . \backslash \backslash$ Version=IA64L-G03RevD $.01 \backslash$ State $=2-\mathrm{A} " \backslash \mathrm{HF}=-301.4687875 \backslash \mathrm{MP} 2=-302.261412 \backslash \mathrm{RMSD}=2.926 \mathrm{e}-09 \backslash \mathrm{Thermal}=$ $0 . \backslash P G=C S[S G(C 2 \mathrm{H} 2 \mathrm{O} 3)] \backslash \backslash @$

\section{$\mathrm{H}-\mathrm{HC}\left(\mathrm{CO}_{2} \mathrm{H}\right)-\mathrm{F}$}

$1 \backslash 1 \backslash G I N C-B A R O S S A 084 \backslash S P \backslash R O M P 2-F C \backslash 6-31 G(d) \backslash C 2 H 3 F 102 \backslash S A N D A L G \backslash 26-J u 1-2005 \backslash$ $0 \backslash \backslash$ \#ROMP2/6-31G* SCF=TIGHT \Cleavage product b3lyp/6-31G** fluoroaceti $\mathrm{c} 1 \_\mathrm{b} 3 \mathrm{p} \backslash \backslash 0,1 \backslash \mathrm{C}, 0,0.493638,-0.907277,0 . \backslash \mathrm{H}, 0,1.115377,-1.073411,0.888308 \backslash$ $\mathrm{H}, \overline{0}, 1.115377,-1.073411,-0.888308 \backslash \mathrm{F}, 0,-0.571901,-1.770392,0 . \backslash \mathrm{C}, 0,0 ., 0.5$ $29688,0 . \backslash 0,0,-1.151137,0.883716,0 . \backslash 0,0,1.058789,1.375031,0 . \backslash \mathrm{H}, 0,0.6933$ $01,2.275913,0 . \backslash \backslash$ Version=IA32L-G03RevC.02 $\backslash$ State $=1-A^{\prime} \backslash \mathrm{HF}=-326.6463023 \backslash \mathrm{MP}$ $2=-327.4227885 \backslash \mathrm{RMSD}=3.817 \mathrm{e}-09 \backslash \mathrm{PG}=\mathrm{CS} \quad[\mathrm{SG}(\mathrm{C} 2 \mathrm{H} 1 \mathrm{~F} 1 \mathrm{O} 2), \mathrm{X}(\mathrm{H} 2)] \backslash \backslash @$

\section{$\mathrm{H}-\cdot \mathrm{C}\left(\mathrm{CO}_{2} \mathrm{H}\right)-\mathrm{F}$}

$1 \backslash 1 \backslash G I N C-B A R O S S A 068 \backslash S P \backslash R O M P 2-F C \backslash 6-31 G(d) \backslash C 2 H 2 F 1 O 2$ ( 2$) \backslash S A N D A L G \backslash 26-J u l-20$

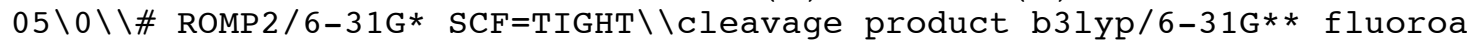

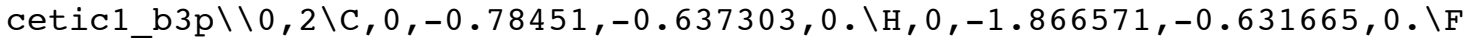
$, 0,-0.1968,-1.82717,0 . \backslash \mathrm{C}, 0,0 ., 0.570211,0 . \backslash 0,0,-0.513336,1.680568,0 . \backslash 0$, $0,1.337707,0.350528,0 . \backslash \mathrm{H}, 0,1.749867,1.229985,0 . \backslash \backslash$ Version=IA32L-G03RevC $.02 \backslash$ State $=2-\mathrm{A} " \backslash \mathrm{HF}=-326.0177543 \backslash \mathrm{MP} 2=-326.7783636 \backslash \mathrm{RMSD}=5.344 \mathrm{e}-09 \backslash \mathrm{PG}=\mathrm{CS} \quad$ [ $\mathrm{SG}(\mathrm{C} 2 \mathrm{H} 2 \mathrm{~F} 1 \mathrm{O} 2)] \backslash \backslash @$ 


\section{$\mathrm{HO}-\mathrm{HC}\left(\mathrm{CO}_{2} \mathrm{H}\right)-\mathrm{NH}_{2}$}

$1 \backslash 1 \backslash G I N C-B A R O S S A 104 \backslash S P \backslash R O M P 2-F C \backslash 6-31 G(d) \backslash C 2 H 5 N 103 \backslash S A N D A L G \backslash 28-J u n-2005 \backslash$ $0 \backslash \backslash$ \#ROMP2 $/ 6-31 \mathrm{G} *$ SCF=TIGHT $\backslash$ hydroxyglycine1 b3lyp/6-31G** $\backslash \backslash 0,1 \backslash \mathrm{N}, 0,-1$. $266603,-1.157469,-0.176466 \backslash C, 0,-0.684501,0.053878,0.374945 \backslash 0,0,-1.2767$ $37,1.23712,-0.091013 \backslash \mathrm{C}, 0,0.824,0.070067,0.049498 \backslash \mathrm{H}, 0,-0.77015,-0.02587$ $5,1.471967 \backslash 0,0,1.423741,1.109262,-0.116062 \backslash 0,0,1.39028,-1.137644,-0.00$ $2299 \backslash \mathrm{H}, 0,0.642649,-1.774564,0.034614 \backslash \mathrm{H}, 0,-1.435477,-1.018952,-1.171963$ $\backslash \mathrm{H}, 0,-2.166406,-1.351811,0.25284 \backslash \mathrm{H}, 0,-0.539663,1.85991,-0.223854 \backslash \backslash$ Vers ion $=I A 32 L-G 03 R e v C .02 \backslash$ State $=1-A \backslash H F=-357.682297 \backslash M P 2=-358.6369511 \backslash$ RMSD $=6$. $812 \mathrm{e}-09 \backslash \mathrm{PG}=\mathrm{C} 01 \quad[\mathrm{X}(\mathrm{C} 2 \mathrm{H} 5 \mathrm{~N} 1 \mathrm{O} 3)] \backslash \backslash @$

\section{$\mathrm{HO}-\cdot \mathrm{C}\left(\mathrm{CO}_{2} \mathrm{H}\right)-\mathrm{NH}_{2}$}

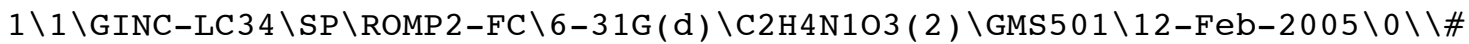
ROMP2 /6-31G* SCF=TIGHT \\hydroxyglycyl_rad9 from hydroxyglutamate romp2 $/ 6-31 \mathrm{G} * / / \mathrm{b} 31 \mathrm{yp} / 6-31 \mathrm{G} * * \backslash \backslash 0,2 \backslash \mathrm{C}, 0,0.74693,-0.083648,-0.00285 \backslash 0,0,1.34775$ $3,-1.170807,0.003289 \backslash 0,0,1.381784,1.132734,0.004957 \backslash \mathrm{H}, 0,2.329905,0.930$ $524,0.01608 \backslash \mathrm{C}, 0,-0.670001,-0.006729,-0.001638 \backslash \mathrm{N}, 0,-1.406835,1.143873,-$ $0.07666 \backslash \mathrm{H}, 0,-0.900202,1.98804,0.149237 \backslash \mathrm{H}, 0,-2.343633,1.090523,0.301376$ $\backslash 0,0,-1.358722,-1.164361,0.002751 \backslash \mathrm{H}, 0,-0.666325,-1.854466,0.008879 \backslash \backslash \mathrm{Ve}$ rsion $=I A 32 L-G 03 R e v C .02 \backslash$ State $=2-A \backslash H F=-357.0710324 \backslash M P 2=-358.0142463 \backslash$ RMSD $=3.062 \mathrm{e}-09 \backslash \mathrm{PG}=\mathrm{C} 01 \quad[\mathrm{X}(\mathrm{C} 2 \mathrm{H} 4 \mathrm{~N} 103)] \backslash \backslash @$

\section{$\mathrm{H}_{2} \mathrm{~N}-\mathrm{HC}\left(\mathrm{CO}_{2} \mathrm{H}\right)-\mathrm{NH}_{2}$}

$1 \backslash 1 \backslash G I N C-B A R O S S A 125 \backslash S P \backslash R O M P 2-F C \backslash 6-31 G(d) \backslash C 2 H 6 N 2 O 2 \backslash S A N D A L G \backslash 27-J u 1-2005 \backslash$ $0 \backslash \backslash \#$ ROMP $2 / 6-31 \mathrm{G} * \mathrm{SCF}=\mathrm{TIGHT} \backslash \backslash \mathrm{cleavage}$ product b3lyp/6-31G** two-aminoc leav-nonrad $1 \backslash \backslash 0,1 \backslash \mathrm{C}, 0,-0.674622,0.047584,0.362352 \backslash \mathrm{H}, 0,-0.742454,0.0294$ $92,1.463631 \backslash \mathrm{N}, 0,-1.187913,-1.251091,-0.100923 \backslash \mathrm{H}, 0,-1.997064,-1.541959$, $0.439591 \backslash \mathrm{H}, 0,-1.497601,-1.136364,-1.065307 \backslash \mathrm{N}, 0,-1.315303,1.192971,-0.2$ $51105 \backslash \mathrm{H}, 0,-2.100017,1.523593,0.301339 \backslash \mathrm{H}, 0,-0.632688,1.945599,-0.317139$ $\backslash \mathrm{C}, 0,0.842753,0.105416,0.053553 \backslash 0,0,1.452849,1.148121,-0.017867 \backslash 0,0,1$. $402725,-1.098746,-0.080577 \backslash \mathrm{H}, 0,0.638952,-1.726522,-0.065793 \backslash \backslash$ Version=I A32L-G03RevC.02\State $=1-\mathrm{A} \backslash \mathrm{HF}=-337.849773 \backslash \mathrm{MP} 2=-338.7914059 \backslash \mathrm{RMSD}=7.075 \mathrm{e}-$ $09 \backslash P G=C 01 \quad[X(C 2 \mathrm{H} 6 \mathrm{~N} 2 \mathrm{O} 2)] \backslash \backslash @$

\section{$\mathrm{H}_{2} \mathrm{~N}-\cdot \mathrm{C}\left(\mathrm{CO}_{2} \mathrm{H}\right)-\mathrm{NH}_{2}(3 \mathrm{~s})$}

$1 \backslash 1 \backslash G I N C-B A R O S S A 148 \backslash S P \backslash R O M P 2-F C \backslash 6-31 G(d) \backslash C 2 H 5 N 2 O 2$ ( 2$) \backslash S A N D A L G \backslash 29-A p r-20$ $05 \backslash 0 \backslash \backslash \#$ ROMP2/6-31G* SCF=TIGHT $\backslash \backslash 2$-aminoglut cleavage product b3lyp/6-3 $1 G * * \backslash \backslash 0,2 \backslash \mathrm{C}, 0,0.662044,0.027842,0.001724 \backslash \mathrm{C}, 0,-0.753001,-0.15091,0.0042$ $52 \backslash 0,0,-1.320317,-1.248935,0.019466 \backslash 0,0,-1.450609,1.038491,-0.019693 \backslash \mathrm{H}$ $, 0,-2.385269,0.781743,-0.01318 \backslash \mathrm{N}, 0,1.271908,1.266358,0.092176 \backslash \mathrm{N}, 0,1.47$ $3045,-1.08763,-0.096172 \backslash \mathrm{H}, 0,2.266322,-1.092567,0.536912 \backslash \mathrm{H}, 0,0.921589,-$ $1.937389,-0.038693 \backslash \mathrm{H}, 0,0.617799,2.034896,0.021673 \backslash \mathrm{H}, 0,2.078032,1.38418$ $6,-0.512781 \backslash \backslash$ Version $=I A 32 \mathrm{~L}-\mathrm{G} 03$ RevC. $02 \backslash$ State $=2-\mathrm{A} \backslash \mathrm{HF}=-337.2452657 \backslash \mathrm{MP} 2=-3$ $38.1732395 \backslash \mathrm{RMSD}=3.250 \mathrm{e}-09 \backslash \mathrm{PG}=\mathrm{C} 01 \quad[\mathrm{X}(\mathrm{C} 2 \mathrm{H} 5 \mathrm{~N} 2 \mathrm{O} 2)] \backslash \backslash @$

\section{$\mathrm{F}-\mathrm{HC}\left(\mathrm{CO}_{2} \mathrm{H}\right)-\mathrm{NH}_{2}$}

$1 \backslash 1 \backslash \mathrm{GINC}-\mathrm{BAROSSA} 089 \backslash \mathrm{SP} \backslash \mathrm{ROMP} 2-\mathrm{FC} \backslash 6-31 \mathrm{G}(\mathrm{d}) \backslash \mathrm{C} 2 \mathrm{H} 4 \mathrm{~F} 1 \mathrm{~N} 1 \mathrm{O} 2 \backslash \mathrm{SANDALG} \backslash 26-\mathrm{Jul}-200$ $5 \backslash 0 \backslash \backslash \#$ ROMP2/6-31G* SCF=TIGHT \Cleavage product b3lyp/6-31G** fluorogl

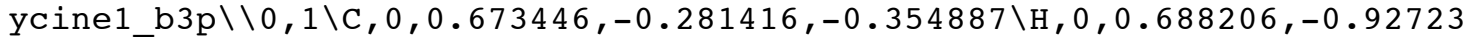
$8,-1.2 \overline{3} 6019 \backslash \mathrm{C}, 0,-0.747455,0.170273,-0.012442 \backslash 0,0,-1.003226,1.139282,0$. $66447 \backslash 0,0,-1.673968,-0.665348,-0.515061 \backslash \mathrm{H}, 0,-2.538982,-0.339354,-0.211$ $319 \backslash \mathrm{N}, 0,1.482474,0.851395,-0.536149 \backslash \mathrm{H}, 0,1.369202,1.507506,0.230924 \backslash \mathrm{H}, 0$ $, 2.458721,0.614834,-0.67015 \backslash F, 0,1.056349,-1.104459,0.738701 \backslash \backslash$ Version=I A32L-G03RevC. 02 \State $=1-A \backslash H F=-381.681326 \backslash M P 2=-382.6238889 \backslash \mathrm{RMSD}=5.046 \mathrm{e}-$ $09 \backslash P G=\mathrm{C} 01 \quad[\mathrm{X}(\mathrm{C} 2 \mathrm{H} 4 \mathrm{~F} 1 \mathrm{~N} 1 \mathrm{O} 2)] \backslash \backslash @$ 


\section{$\mathrm{F}-\cdot \mathrm{C}\left(\mathrm{CO}_{2} \mathrm{H}\right)-\mathrm{NH}_{2}$}

$1 \backslash 1 \backslash G I N C-B A R O S S A 125 \backslash S P \backslash R O M P 2-F C \backslash 6-31 G(d) \backslash C 2 H 3 F 1 N 1 O 2(2) \backslash S A N D A L G \backslash 27-J u 1-$ $2005 \backslash 0 \backslash \backslash \#$ ROMP $2 / 6-31 \mathrm{G}$ * SCF=TIGHT \\Cleavage product b3lyp/6-31G** fluor

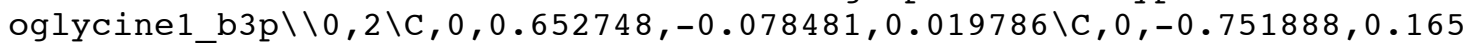
$894,0.005 \overline{7} 28 \backslash 0,0,-1.218073,1.308242,0.022925 \backslash 0,0,-1.513947,-0.96213,-0$ $.015034 \backslash \mathrm{H}, 0,-2.431688,-0.646916,-0.010681 \backslash \mathrm{N}, 0,1.55675,0.935917,-0.0928$ $39 \backslash \mathrm{H}, 0,1.135376,1.849307,0.025197 \backslash \mathrm{H}, 0,2.453708,0.801367,0.356247 \backslash \mathrm{F}, 0,1$ $.155151,-1.316505,0.006988 \backslash \backslash$ Version=IA32L-G03RevC . 02 $\backslash$ State $=2-A \backslash H F=-381$ $.0566943 \backslash \mathrm{MP} 2=-381.9872276 \backslash \mathrm{RMSD}=2.183 \mathrm{e}-09 \backslash \mathrm{PG}=\mathrm{C} 01 \quad[\mathrm{X}(\mathrm{C} 2 \mathrm{H} 3 \mathrm{~F} 1 \mathrm{~N} 1 \mathrm{O} 2)] \backslash \backslash \mathrm{e}$

\section{$\mathrm{HO}-\mathrm{HC}\left(\mathrm{CO}_{2} \mathrm{H}\right)-\mathrm{OH}$}

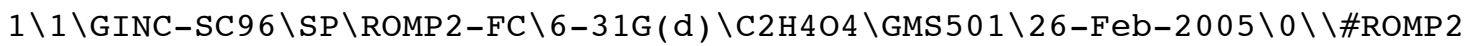
/6-31G* SCF=TIGHT $\backslash \backslash 23 \mathrm{~S}$ dihydroxyacetic acid romp $2 / 6-31 \mathrm{G} * / / \mathrm{b} 31 \mathrm{yp} / 6-31 \mathrm{G}($ $\mathrm{d}, \mathrm{p}) \backslash \backslash 0,1 \backslash \mathrm{C}, 0,0.693928,-0.219972,0.374686 \backslash \mathrm{H}, 0,0.744485,-0.678566,1.370$ $493 \backslash \mathrm{C}, 0,-0.75397,0.143604,0.018974 \backslash 0,0,-1.045483,1.173045,-0.54944 \backslash 0,0$ $,-1.635339,-0.797844,0.382599 \backslash \mathrm{H}, 0,-2.51119,-0.498674,0.082321 \backslash 0,0,1.08$ $5629,-1.129514,-0.632346 \backslash \mathrm{H}, 0,2.022255,-1.320877,-0.482578 \backslash 0,0,1.468037$ $, 0.933801,0.417318 \backslash \mathrm{H}, 0,1.121941,1.520422,-0.277252 \backslash \backslash$ Version=Al64T-G03R evC. $02 \backslash$ State $=1-A \backslash H F=-377.5125308 \backslash M P 2=-378.4778289 \backslash \mathrm{RMSD}=5.884 \mathrm{e}-09 \backslash \mathrm{PG}=\mathrm{C} 0$ $1[\mathrm{X}(\mathrm{C} 2 \mathrm{H} 4 \mathrm{O} 4)] \backslash \backslash @$

\section{$\mathrm{HO}-\cdot \mathrm{C}\left(\mathrm{CO}_{2} \mathrm{H}\right)-\mathrm{OH}(3 \mathrm{f})$}

$1 \backslash 1 \backslash G I N C-L C 18 \backslash S P \backslash R O M P 2-F C \backslash 6-31 G(d) \backslash C 2 H 3 O 4$ ( 2$) \backslash G M S 501 \backslash 24-F e b-2005 \backslash 0 \backslash \backslash \# R O$ MP2/6-31G* SCF=TIGHT \CS dihydroxyacetic acid radical romp $2 / 6-31 \mathrm{G} * / / \mathrm{b} 3$

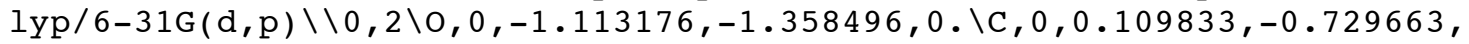
$0 . \backslash 0,0,1.19646,-1.321465,0 . \backslash C, 0,0 ., 0.685771,0 . \backslash 0,0,1.101474,1.437276,0$ $. \backslash 0,0,-1.14702,1.373473,0 . \backslash \mathrm{H}, 0,-0.931067,-2.310554,0 . \backslash \mathrm{H}, 0,1.84087,0.80$ $0312,0 . \backslash \mathrm{H}, 0,-1.870704,0.7273,0 . \backslash \backslash$ Version=IA32L-G03RevC.02 $\backslash$ state $=2-\mathrm{A} " \backslash \mathrm{H}$ $\mathrm{F}=-376.8931509 \backslash \mathrm{MP} 2=-377.8504176 \backslash \mathrm{RMSD}=7.570 \mathrm{e}-09 \backslash \mathrm{PG}=\mathrm{CS} \quad[\mathrm{SG}(\mathrm{C} 2 \mathrm{H} 3 \mathrm{O} 4)] \backslash \backslash @$

\section{$\mathrm{O}=\mathrm{HC}\left(\mathrm{CO}_{2} \mathrm{H}\right)$}

$1 \backslash 1 \backslash G I N C-B A R O S S A 063 \backslash S P \backslash R O M P 2-F C \backslash 6-31 G(d) \backslash C 2 H 2 O 3 \backslash S A N D A L G \backslash 28-J u n-2005 \backslash 0 \backslash$

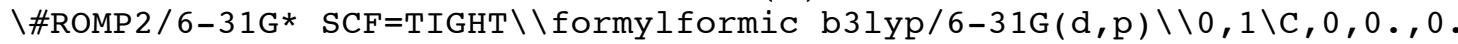
$584127,0 . \backslash \mathrm{C}, 0,-0.75137,-0.752687,0 . \backslash \mathrm{H}, 0,-1.852485,-0.675527,0 . \backslash 0,0,-0$. $578795,1.641904,0 . \backslash 0,0,-0.144285,-1.801869,0 . \backslash 0,0,1.329466,0.436391,0$. $\backslash \mathrm{H}, 0,1.509617,-0.52452,0 . \backslash \backslash$ Version=IA32L-G03RevC.02 $\backslash$ State $=1-A^{\prime} \backslash \mathrm{HF}=-301$ $.4758172 \backslash M P 2=-302.2562192 \backslash \mathrm{RMSD}=5.320 \mathrm{e}-09 \backslash \mathrm{PG}=\mathrm{CS} \quad[\mathrm{SG}(\mathrm{C} 2 \mathrm{H} 2 \mathrm{O} 3)] \backslash \backslash @$

\section{$\mathrm{O}=\cdot \mathrm{C}\left(\mathrm{CO}_{2} \mathrm{H}\right)(3 \mathrm{e})$}

$1 \backslash 1 \backslash G I N C-S C 64 \backslash S P \backslash R O M P 2-F C \backslash 6-31 G(d) \backslash C 2 H 103(2) \backslash G M S 501 \backslash 27-F e b-2005 \backslash 0 \backslash \backslash \# R O$ MP2/6-31G* SCF=TIGHT \formylformic radical romp2/6-31G*//b3lyp/6-31G(d ,p) $\backslash \backslash 0,2 \backslash \mathrm{C}, 0,0.472635,0.114325,0.02283 \backslash 0,0,0.963847,1.21624,-0.041598 \backslash$ $0,0,1.128187,-1.057177,-0.043558 \backslash \mathrm{H}, 0,2.069882,-0.849059,-0.17744 \backslash \mathrm{C}, 0,-$ $0.971281,-0.098853,0.404952 \backslash 0,0,-1.976784,-0.064534,-0.2135 \backslash \backslash$ Version $=\mathrm{A}$ $164 \mathrm{~T}-\mathrm{G} 03 \mathrm{RevC} .02 \backslash \mathrm{State}=2-\mathrm{A} \backslash \mathrm{HF}=-300.848153 \backslash \mathrm{MP} 2=-301.6192816 \backslash \mathrm{RMSD}=9.793 \mathrm{e}-$ $09 \backslash P G=C 01 \quad[X(\mathrm{C} 2 \mathrm{H} 103)] \backslash \backslash @$

\section{$1 \mathrm{c}$}

$1 \backslash 1 \backslash \mathrm{GINC}-\mathrm{AC} 27 \backslash \mathrm{SP} \backslash \mathrm{ROMP} 2-\mathrm{FC} \backslash 6-31 \mathrm{G}(\mathrm{d}) \backslash \mathrm{C} 5 \mathrm{H} 8 \mathrm{O} 4 \mathrm{~S} 1 \backslash \mathrm{GMS} 501 \backslash 23-\mathrm{Feb}-2006 \backslash 0 \backslash \backslash$ \#rom p2/6-31G* scf=tight $\backslash$ Xtal thiolgutarqate b3lyp/6-31G** $\backslash \backslash 0,1 \backslash C, 0,0.7918$ $63,-0.33608,-0.13791 \backslash C, 0,1.224174,1.116277,-0.034897 \backslash 0,0,1.389963,1.71$ $4824,1.007899 \backslash \mathrm{C}, 0,-0.149204,-0.745586,1.001142 \backslash \mathrm{C}, 0,-1.468773,0.035048$, $1.036318 \backslash \mathrm{C}, 0,-2.368842,-0.223585,-0.152482 \backslash 0,0,-2.116667,-0.939194,-1$. $098846 \backslash 0,0,-3.536585,0.452965,-0.045485 \backslash 0,0,1.417509,1.679909,-1.24560$ $6 \backslash \mathrm{H}, 0,1.739277,2.582596,-1.080254 \backslash \mathrm{H}, 0,0.318616,-0.50839,-1.106048 \backslash \mathrm{H}, 0$, 
$-0.354315,-1.814357,0.901408 \backslash \mathrm{H}, 0,0.365216,-0.589255,1.953961 \backslash \mathrm{H}, 0,-1.29$ $3018,1.114602,1.109668 \backslash \mathrm{H}, 0,-4.050743,0.234883,-0.841531 \backslash \mathrm{H}, 0,-2.036418$, $-0.224978,1.937609 \backslash \mathrm{S}, 0,2.320955,-1.381415,-0.195192 \backslash \mathrm{H}, 0,2.767045,-1.03$ $695,1.031533 \backslash \backslash$ Version=IA64L-G03RevD. 01 \State=1-A \HF=-890.995765 \MP2=-8 $92.4689081 \backslash \mathrm{RMSD}=8.696 \mathrm{e}-09 \backslash \mathrm{Thermal}=0 . \backslash \mathrm{PG}=\mathrm{C} 01 \quad[\mathrm{X}(\mathrm{C} 5 \mathrm{H} 804 \mathrm{~S} 1)] \backslash \backslash @$

\section{2c}

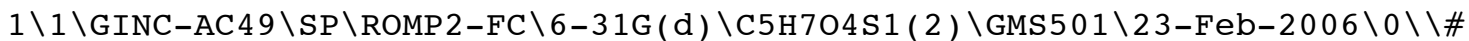

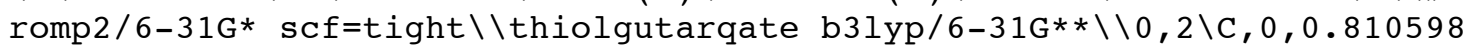
$,-0.334837,-0.157185 \backslash \mathrm{C}, 0,1.2719,1.109656,-0.05946 \backslash 0,0,1.444071,1.70437$ $4,0.983366 \backslash \mathrm{C}, 0,-0.188897,-0.690833,0.970645 \backslash \mathrm{C}, 0,-1.432468,0.127897,0.8$ $91824 \backslash \mathrm{C}, 0,-2.460641,-0.209317,-0.074067 \backslash 0,0,-2.381366,-1.128795,-0.88 \backslash$ $\mathrm{O}, 0,-3.542695,0.611855,-0.008919 \backslash \mathrm{O}, 0,1.486648,1.659564,-1.271361 \backslash \mathrm{H}, 0,1$ $.822774,2.558168,-1.1119 \backslash \mathrm{H}, 0,0.350954,-0.511661,-1.13155 \backslash \mathrm{H}, 0,-0.432825$ $,-1.751053,0.859099 \backslash \mathrm{H}, 0,0.302795,-0.531376,1.933571 \backslash \mathrm{H}, 0,-1.559564,1.01$ $3359,1.504828 \backslash \mathrm{H}, 0,-4.156229,0.299667,-0.694375 \backslash \mathrm{S}, 0,2.304215,-1.426887$, $-0.152287 \backslash \mathrm{H}, 0,2.748431,-1.038288,1.061695 \backslash \backslash$ Version=IA64L-G03RevD.01\St ate $=2-\mathrm{A} \backslash \mathrm{HF}=-890.3690124 \backslash \mathrm{MP} 2=-891.8210819 \backslash \mathrm{RMSD}=9.384 \mathrm{e}-09 \backslash \mathrm{Thermal}=0 . \backslash \mathrm{PG}=$ $\mathrm{C} 01[\mathrm{X}(\mathrm{C} 5 \mathrm{H} 7 \mathrm{O} 4 \mathrm{~S} 1)] \backslash \backslash @$

\section{C3-radical derived from 1c}

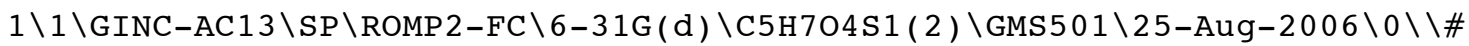

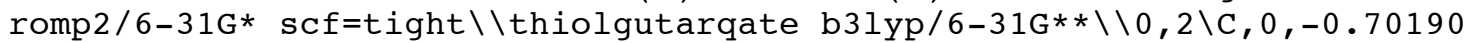
$3,0.026798,-0.114492 \backslash \mathrm{C}, 0,-1.959118,-0.766951,0.188461 \backslash 0,0,-2.512493,-0$ $.798031,1.2667 \backslash \mathrm{C}, 0,0.230439,0.050817,1.023993 \backslash \mathrm{C}, 0,1.651231,0.487713,0$. $886765 \backslash \mathrm{C}, 0,2.478302,-0.30408,-0.117119 \backslash 0,0,2.054847,-0.988799,-1.02223$ $7 \backslash 0,0,3.80123,-0.121088,0.099924 \backslash 0,0,-2.405878,-1.434035,-0.894182 \backslash \mathrm{H}, 0$ $,-3.225492,-1.881272,-0.622348 \backslash \mathrm{H}, 0,-0.230581,-0.331303,-1.030516 \backslash \mathrm{H}, 0,-$ $0.197571,-0.048614,2.015943 \backslash \mathrm{H}, 0,2.167178,0.464229,1.85004 \backslash \mathrm{H}, 0,4.262774$ $,-0.619304,-0.596314 \backslash \mathrm{H}, 0,1.704821,1.536708,0.544835 \backslash \mathrm{S}, 0,-1.270073,1.78$ $7397,-0.59739 \backslash \mathrm{H}, 0,-1.855315,2.051053,0.589303 \backslash \backslash$ Version=IA64L-G03RevD.0 $1 \backslash$ State $=2-A \backslash H F=-890.361583 \backslash M P 2=-891.8122843 \backslash \mathrm{RMSD}=7.462 \mathrm{e}-09 \backslash \mathrm{Thermal}=0 . \backslash$ $\mathrm{PG}=\mathrm{C} 01 \quad[\mathrm{X}(\mathrm{C} 5 \mathrm{H} 704 \mathrm{~S} 1)] \backslash \backslash @$

\section{TS: $2 c \rightarrow 3 c^{*}$}

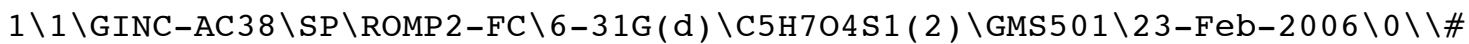

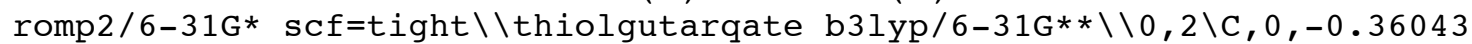
$2,-0.734033,1.181428 \backslash \mathrm{C}, 0,1.091873,-0.295157,-0.406591 \backslash \mathrm{H}, 0,0.397113,-0$. $578845,-1.188498 \backslash \mathrm{S}, 0,2.45759,-1.374639,-0.2074 \backslash \mathrm{H}, 0,3.179541,-0.490457$, $0.518733 \backslash \mathrm{C}, 0,1.325669,1.13889,-0.179979 \backslash 0,0,2.254788,1.608742,0.456796$ $\backslash \mathrm{O}, 0,0.347857,1.903599,-0.721193 \backslash \mathrm{H}, 0,0.557563,2.821204,-0.479072 \backslash \mathrm{C}, 0,-$ $1.473911,0.052772,0.998926 \backslash \mathrm{C}, 0,-2.464174,-0.314087,-0.015568 \backslash 0,0,-2.35$ $7267,-1.242899,-0.801372 \backslash 0,0,-3.542869,0.511334,-0.010225 \backslash \mathrm{H}, 0,-0.38161$ $7,-1.751828,0.808801 \backslash \mathrm{H}, 0,0.322388,-0.528671,1.998728 \backslash \mathrm{H}, 0,-1.604368,0.9$ $96832,1.515708 \backslash \mathrm{H}, 0,-4.126279,0.189473,-0.717354 \backslash \backslash$ Version=IA64L-G03RevD $.01 \backslash$ State $=2-A \backslash H F=-890.3072549 \backslash M P 2=-891.7807069 \backslash \mathrm{RMSD}=9.843 \mathrm{e}-09 \backslash \mathrm{Thermal}=$ $0 . \backslash \mathrm{PG}=\mathrm{C} 01 \quad[\mathrm{X}(\mathrm{C} 5 \mathrm{H} 704 \mathrm{~S} 1)] \backslash \backslash @$

\section{$\mathrm{TS}: 3 \mathrm{c}^{*} \rightarrow \mathbf{4 c}$}

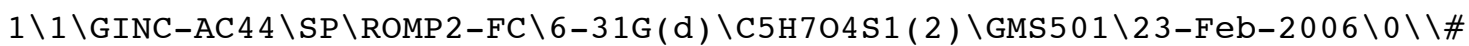

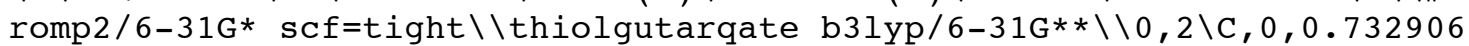
$,-0.975045,0.452071 \backslash \mathrm{C}, 0,-0.594199,0.292768,-0.58858 \backslash \mathrm{C}, 0,-1.863035,-0.3$ $54906,-0.197825 \backslash \mathrm{H}, 0,-1.302695,1.955947,0.838827 \backslash 0,0,-2.602305,0.01833$, $0.693812 \backslash \mathrm{O}, 0,-2.084856,-1.492412,-0.904967 \backslash \mathrm{H}, 0,-2.904144,-1.875184,-0$. $548548 \backslash \mathrm{H}, 0,-0.221229,0.049244,-1.577939 \backslash \mathrm{S}, 0,-0.421759,1.999107,-0.1841$ $9 \backslash \mathrm{C}, 0,1.998582,-0.353019,-0.037684 \backslash 0,0,2.7128,0.392658,0.596103 \backslash 0,0,2$. 
$264188,-0.703445,-1.324199 \backslash \mathrm{C}, 0,0.496753,-0.940046,1.823569 \backslash \mathrm{H}, 0,0.38859$ $4,-1.831115,-0.121895 \backslash \mathrm{H}, 0,-0.291533,-1.532095,2.273221 \backslash \mathrm{H}, 0,1.044256,-0$ $.243545,2.447873 \backslash \mathrm{H}, 0,3.090223,-0.248526,-1.559805 \backslash \backslash$ Version=IA64L-G03Re $\mathrm{VD} .01 \backslash \mathrm{State}=2-\mathrm{A} \backslash \mathrm{HF}=-890.2967519 \backslash \mathrm{MP} 2=-891.7726792 \backslash \mathrm{RMSD}=3.472 \mathrm{e}-09 \backslash \mathrm{Therma}$ $1=0 . \backslash P G=\operatorname{C} 01 \quad[X(C 5 H 704 \mathrm{~S} 1)] \backslash \backslash @$

\section{4c}

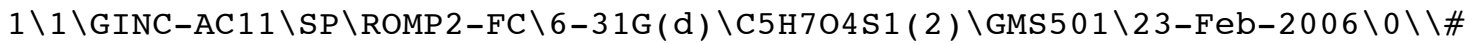

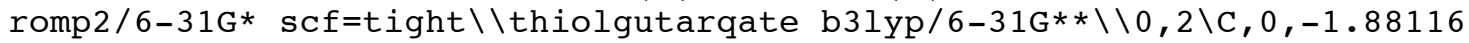
$6,-0.38517,-0.132216 \backslash \mathrm{C}, 0,-0.478707,0.114078,-0.451172 \backslash \mathrm{S}, 0,-0.338566,1$. $929337,-0.218274 \backslash \mathrm{H}, 0,-0.869881,1.942917,1.020213 \backslash \mathrm{O}, 0,-2.594014,0.01006$ $1,0.761973 \backslash \mathrm{C}, 0,0.567472,-0.718038,0.368241 \backslash \mathrm{C}, 0,1.993543,-0.399405,-0.0$ $82042 \backslash 0,0,2.938874,-0.221132,0.648572 \backslash 0,0,2.099095,-0.388471,-1.432509$ $\backslash \mathrm{C}, 0,0.425195,-0.601947,1.846107 \backslash \mathrm{O}, 0,-2.22633,-1.401749,-0.95601 \backslash \mathrm{H}, 0,-$ $3.106681,-1.701807,-0.670151 \backslash \mathrm{H}, 0,-0.28684,-0.055574,-1.51094 \backslash \mathrm{H}, 0,0.399$ $381,-1.764958,0.049383 \backslash \mathrm{H}, 0,-0.548557,-0.709371,2.306775 \backslash \mathrm{H}, 0,1.301228,-$ $0.438739,2.45861 \backslash \mathrm{H}, 0,3.029386,-0.188633,-1.631226 \backslash \backslash$ Version=IA64L-G03Re $\mathrm{VD} .01 \backslash$ State $=2-\mathrm{A} \backslash \mathrm{HF}=-890.3522126 \backslash \mathrm{MP} 2=-891.8065922 \backslash \mathrm{RMSD}=9.289 \mathrm{e}-09 \backslash \mathrm{Therma}$ $1=0 . \backslash \mathrm{PG}=\mathrm{C} 01 \quad[\mathrm{X}(\mathrm{C} 5 \mathrm{H} 704 \mathrm{~S} 1)] \backslash \backslash @$

\section{$5 c$}

$1 \backslash 1 \backslash G I N C-A C 41 \backslash S P \backslash R O M P 2-F C \backslash 6-31 G(d) \backslash C 5 H 804 S 1 \backslash G M S 501 \backslash 23-F e b-2006 \backslash 0 \backslash \backslash \#$ rom

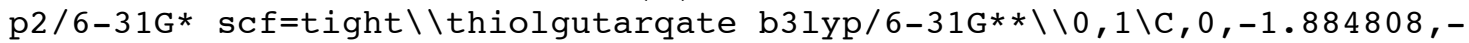
$0.351661,-0.226348 \backslash \mathrm{C}, 0,-0.48039,0.192792,-0.454624 \backslash \mathrm{S}, 0,-0.345043,1.946$ $57,0.085879 \backslash \mathrm{H}, 0,-1.164314,1.816666,1.148368 \backslash \mathrm{C}, 0,0.564271,-0.764915,0.1$ $94041 \backslash \mathrm{C}, 0,1.987419,-0.351153,-0.167854 \backslash 0,0,2.933717,-0.372435,0.583997$ $\backslash 0,0,2.100039,0.005541,-1.471737 \backslash C, 0,0.419536,-0.935086,1.70728 \backslash 0,0,-2$ $.665012,0.003029,0.627538 \backslash 0,0,-2.148484,-1.359292,-1.089966 \backslash \mathrm{H}, 0,-3.034$ $33,-1.691922,-0.864072 \backslash \mathrm{H}, 0,-0.298593,0.206944,-1.529916 \backslash \mathrm{H}, 0,0.414609,-$ $1.738777,-0.296469 \backslash \mathrm{H}, 0,-0.570501,-1.319308,1.965032 \backslash \mathrm{H}, 0,0.566722,0.018$ $645,2.219725 \backslash \mathrm{H}, 0,3.034005,0.237373,-1.60932 \backslash \mathrm{H}, 0,1.174843,-1.629352,2.0$ $78949 \backslash \backslash$ Version=IA64L-G03RevD.01\State $=1-A \backslash H F=-890.9891783 \backslash \mathrm{MP} 2=-892.467$ $7628 \backslash \mathrm{RMSD}=5.714 \mathrm{e}-09 \backslash \mathrm{Thermal}=0 . \backslash \mathrm{PG}=\mathrm{C} 01 \quad[\mathrm{X}(\mathrm{C} 5 \mathrm{H} 804 \mathrm{~S} 1)] \backslash \backslash @$

\section{$1 d$}

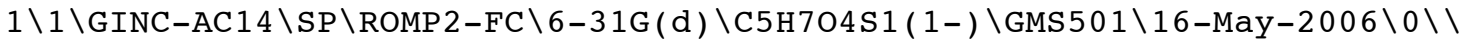

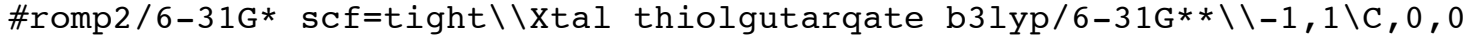
$.762867,-0.392984,-0.24331 \backslash \mathrm{C}, 0,1.256509,0.957593,0.187895 \backslash 0,0,1.336387$ $, 1.384117,1.332876 \backslash \mathrm{C}, 0,-0.197118,-0.991679,0.79029 \backslash \mathrm{C}, 0,-1.475485,-0.15$ $7859,1.104811 \backslash \mathrm{C}, 0,-2.408191,-0.057206,-0.069799 \backslash 0,0,-3.231485,-0.88593$ $5,-0.410403 \backslash 0,0,-2.236649,1.092398,-0.783177 \backslash 0,0,1.625576,1.759038,-0$. $858794 \backslash \mathrm{H}, 0,1.990517,2.546354,-0.423737 \backslash \mathrm{H}, 0,0.258556,-0.275616,-1.21040$ $8 \backslash \mathrm{H}, 0,-0.484891,-1.98977,0.447752 \backslash \mathrm{H}, 0,0.355039,-1.113271,1.726029 \backslash \mathrm{H}, 0$, $-1.177893,0.838727,1.438685 \backslash \mathrm{H}, 0,-2.828687,1.003327,-1.548668 \backslash \mathrm{H}, 0,-2.03$ $1932,-0.653667,1.90689 \backslash \mathrm{S}, 0,2.271073,-1.456264,-0.449993 \backslash \backslash$ Version=IA64L

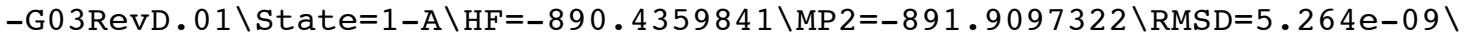
Thermal $=0 . \backslash \mathrm{PG}=\mathrm{C} 01 \quad[\mathrm{X}(\mathrm{C} 5 \mathrm{H} 704 \mathrm{~S} 1)] \backslash \backslash @$

\section{2d}

$1 \backslash 1 \backslash G I N C-A C 17 \backslash S P \backslash R O M P 2-F C \backslash 6-31 G(d) \backslash C 5 H 6 O 4 S 1(1-, 2) \backslash G M S 501 \backslash 16-M a y-2006 \backslash 0$

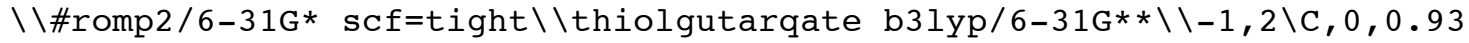
$3913,-0.369889,-0.242247 \backslash \mathrm{C}, 0,1.520813,0.996494,-0.006013 \backslash 0,0,1.572622$, $1.608429,1.047037 \backslash \mathrm{C}, 0,-0.255804,-0.626375,0.784863 \backslash \mathrm{C}, 0,-1.39033,0.2927$ $67,0.576204 \backslash C, 0,-2.602097,-0.112103,-0.051873 \backslash 0,0,-2.912792,-1.236873$, $-0.458232 \backslash 0,0,-3.525072,0.922853,-0.176737 \backslash 0,0,2.027897,1.553718,-1.14$ $8289 \backslash \mathrm{H}, 0,2.421857,2.388238,-0.845542 \backslash \mathrm{H}, 0,0.524829,-0.402897,-1.256001 \backslash$ 
$\mathrm{H}, 0,-0.568576,-1.663662,0.65849 \backslash \mathrm{H}, 0,0.19147,-0.497409,1.774416 \backslash \mathrm{H}, 0,-1$. $279142,1.343953,0.822452 \backslash \mathrm{H}, 0,-4.282349,0.48606,-0.596633 \backslash \mathrm{S}, 0,2.278231$, $-1.595291,-0.064563 \backslash \backslash$ Version=IA64L-G03RevD.01\State $=2-\mathrm{A} \backslash \mathrm{HF}=-889.809475$ $9 \backslash \mathrm{MP} 2=-891.2653911 \backslash \mathrm{RMSD}=8.865 \mathrm{e}-09 \backslash \mathrm{Thermal}=0 . \backslash \mathrm{PG}=\mathrm{C} 01 \quad[\mathrm{X}(\mathrm{C} 5 \mathrm{H} 6 \mathrm{O} 4 \mathrm{~S} 1)] \backslash \backslash @$

\section{C3-radical derived from 1d}

$1 \backslash 1 \backslash G I N C-A C 22 \backslash S P \backslash R O M P 2-F C \backslash 6-31 G(d) \backslash C 5 H 6 O 4 S 1(1-, 2) \backslash G M S 501 \backslash 25-A u g-2006 \backslash 0$

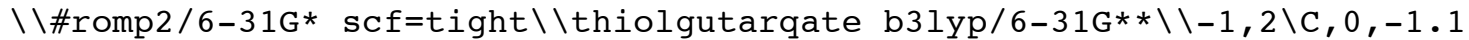
$16731,-0.29845,0.660952 \backslash \mathrm{C}, 0,-1.518482,0.98014,-0.014279 \backslash 0,0,-1.004689$, $1.539566,-0.970655 \backslash \mathrm{C}, 0,0.326168,-0.626805,0.681262 \backslash \mathrm{C}, 0,1.230199,-0.507$ $718,-0.519614 \backslash \mathrm{C}, 0,2.597835,-0.043585,-0.080008 \backslash 0,0,3.569771,-0.744316$, $0.139275 \backslash 0,0,2.671425,1.308569,0.097528 \backslash 0,0,-2.623616,1.540478,0.57624$ $9 \backslash \mathrm{H}, 0,-2.833092,2.293398,-0.000114 \backslash \mathrm{H}, 0,-1.513744,-0.276139,1.679208 \backslash \mathrm{H}$, $0,0.624639,-1.356818,1.430937 \backslash \mathrm{H}, 0,0.802959,0.212775,-1.219778 \backslash \mathrm{H}, 0,3.57$ $4847,1.458035,0.422246 \backslash \mathrm{H}, 0,1.365257,-1.471823,-1.027147 \backslash \mathrm{S}, 0,-2.002371$, $-1.689706,-0.274651 \backslash \backslash$ Version=IA64L-G03RevD .01 \State=2-A \HF=-889.803237 $6 \backslash \mathrm{MP} 2=-891.2551402 \backslash \mathrm{RMSD}=5.080 \mathrm{e}-09 \backslash \mathrm{Thermal}=0 . \backslash \mathrm{PG}=\mathrm{C} 01 \quad[\mathrm{X}(\mathrm{C} 5 \mathrm{H} 6 \mathrm{O} 4 \mathrm{~S} 1)] \backslash \backslash \mathrm{a}$

\section{TS:2d $\rightarrow 3 d^{*}$}

$1 \backslash 1 \backslash G I N C-A C 14 \backslash S P \backslash R O M P 2-F C \backslash 6-31 G(d) \backslash C 5 H 6 O 4 S 1(1-, 2) \backslash G M S 501 \backslash 16-M a y-2006 \backslash 0$

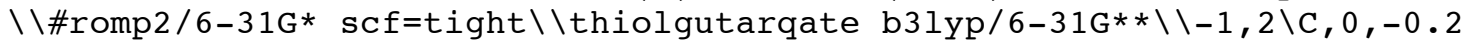
$27268,-0.753192,0.976038 \backslash \mathrm{C}, 0,1.238448,-0.330151,-0.415723 \backslash \mathrm{H}, 0,0.606941$ $,-0.525356,-1.280522 \backslash \mathrm{S}, 0,2.582595,-1.38457,-0.160543 \backslash \mathrm{C}, 0,1.360168,1.11$ $5408,-0.128904 \backslash 0,0,1.995085,1.673845,0.749658 \backslash 0,0,0.572072,1.859446,-0$ $.980489 \backslash \mathrm{H}, 0,0.656399,2.765061,-0.640377 \backslash \mathrm{C}, 0,-1.375844,0.040745,0.87646$ $7 \backslash \mathrm{C}, 0,-2.470792,-0.320231,0.02749 \backslash 0,0,-2.601468,-1.329592,-0.667608 \backslash 0$, $0,-3.501994,0.613264,0.049702 \backslash \mathrm{H}, 0,-0.306757,-1.783653,0.646145 \backslash \mathrm{H}, 0,0.4$ $43223,-0.588445,1.813047 \backslash \mathrm{H}, 0,-1.425747,1.009507,1.363025 \backslash \mathrm{H}, 0,-4.153408$ $, 0.224832,-0.554925 \backslash \backslash$ Version=IA64L-G03RevD . 01 \State=2-A \HF=-889.769743 $2 \backslash \mathrm{MP} 2=-891.2571276 \backslash \mathrm{RMSD}=4.372 \mathrm{e}-09 \backslash \mathrm{Thermal}=0 . \backslash \mathrm{PG}=\mathrm{C} 01 \quad[\mathrm{X}(\mathrm{C} 5 \mathrm{H} 604 \mathrm{~S} 1)] \backslash \backslash @$

\section{TS: $3 d^{*} \rightarrow \mathbf{4 d}$}

$1 \backslash 1 \backslash G I N C-A C 11 \backslash S P \backslash R O M P 2-F C \backslash 6-31 G(d) \backslash C 5 H 6 O 4 S 1(1-, 2) \backslash G M S 501 \backslash 16-M a y-2006 \backslash 0$

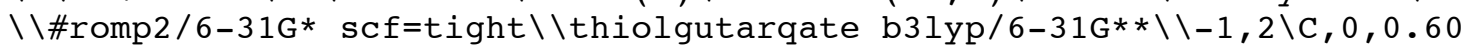
$5423,-0.951635,0.254504 \backslash \mathrm{C}, 0,-0.541568,0.406122,-0.43316 \backslash \mathrm{C}, 0,-1.860833$, $-0.235542,-0.141785 \backslash 0,0,-2.513824,-0.234389,0.882098 \backslash 0,0,-2.312296,-0$. $963431,-1.231341 \backslash \mathrm{H}, 0,-3.142153,-1.354618,-0.911768 \backslash \mathrm{H}, 0,-0.294706,0.248$ $627,-1.485059 \backslash \mathrm{S}, 0,-0.302152,2.047878,0.12797 \backslash \mathrm{C}, 0,1.938718,-0.400593,-0$ $.104824 \backslash 0,0,2.756432,0.098949,0.643015 \backslash 0,0,2.190665,-0.485797,-1.45211$ $3 \backslash \mathrm{C}, 0,0.434466,-1.271718,1.639362 \backslash \mathrm{H}, 0,0.297322,-1.730799,-0.446101 \backslash \mathrm{H}, 0$ $,-0.420658,-1.858094,1.956668 \backslash \mathrm{H}, 0,0.947855,-0.676748,2.385321 \backslash \mathrm{H}, 0,3.02$ $1714,0.003112,-1.564425 \backslash \backslash$ Version=IA64L-G03RevD.01 $\backslash$ State $=2-A \backslash H F=-889.76$ $39468 \backslash \mathrm{MP} 2=-891.2302109 \backslash \mathrm{RMSD}=5.022 \mathrm{e}-09 \backslash \mathrm{Thermal}=0 . \backslash \mathrm{PG}=\mathrm{C} 01 \quad[\mathrm{X}(\mathrm{C} 5 \mathrm{H} 6 \mathrm{O} 4 \mathrm{~S} 1)] \backslash$ $\backslash$ @

\section{4d}

$1 \backslash 1 \backslash G I N C-A C 11 \backslash S P \backslash R O M P 2-F C \backslash 6-31 G(d) \backslash C 5 H 6 O 4 S 1(1-, 2) \backslash G M S 501 \backslash 16-M a y-2006 \backslash 0$

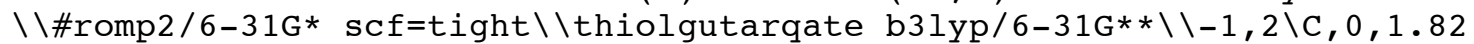
$5618,0.329894,-0.384801 \backslash \mathrm{C}, 0,0.472368,-0.308318,-0.239953 \backslash \mathrm{S}, 0,0.516927$, $-1.404927,1.250436 \backslash 0,0,2.198782,1.425127,0.009894 \backslash \mathrm{C}, 0,-0.658271,0.7643$ $42,-0.145953 \backslash \mathrm{C}, 0,-2.012691,0.115344,-0.375435 \backslash 0,0,-3.015067,0.244012,0$ $.297238 \backslash 0,0,-2.050021,-0.61411,-1.53081 \backslash \mathrm{C}, 0,-0.648392,1.555301,1.12033$ $9 \backslash 0,0,2.70273,-0.469325,-1.07268 \backslash \mathrm{H}, 0,3.543738,0.011933,-1.011834 \backslash \mathrm{H}, 0,0$ $.306753,-0.907209,-1.141025 \backslash \mathrm{H}, 0,-0.531811,1.441844,-1.01681 \backslash \mathrm{H}, 0,0.2859$ $63,1.992734,1.440361 \backslash \mathrm{H}, 0,-1.494571,1.506657,1.792113 \backslash \mathrm{H}, 0,-2.944082,-0$. $992122,-1.544094 \backslash \backslash$ Version=IA64L-G03RevD.01\State=2-A $\backslash H F=-889.7966406 \backslash M$ $\mathrm{P} 2=-891.2487478 \backslash \mathrm{RMSD}=9.711 \mathrm{e}-09 \backslash \mathrm{Thermal}=0 . \backslash \mathrm{PG}=\mathrm{C} 01 \quad[\mathrm{X}(\mathrm{C} 5 \mathrm{H} 604 \mathrm{~S} 1)] \backslash \backslash @$ 
5d

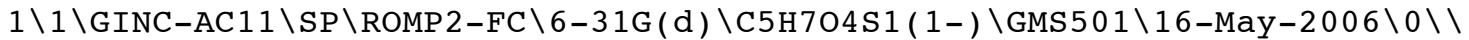

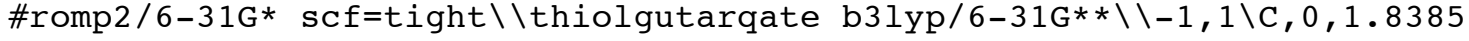
$7,0.27499,-0.414566 \backslash \mathrm{C}, 0,0.483496,-0.340019,-0.19767 \backslash \mathrm{S}, 0,0.540221,-1.27$ $1436,1.403422 \backslash \mathrm{C}, 0,-0.65909,0.717857,-0.286695 \backslash \mathrm{C}, 0,-2.000697,0.013854,-$ $0.396491 \backslash 0,0,-3.002098,0.24088,0.251957 \backslash 0,0,-2.031743,-0.904758,-1.408$ $578 \backslash \mathrm{C}, 0,-0.690082,1.744219,0.847195 \backslash 0,0,2.220664,1.404105,-0.141333 \backslash 0$, $0,2.710013,-0.596557,-1.017418 \backslash \mathrm{H}, 0,3.552876,-0.114827,-1.01024 \backslash \mathrm{H}, 0,0.3$ $30495,-1.053001,-1.012344 \backslash \mathrm{H}, 0,-0.541076,1.245799,-1.249931 \backslash \mathrm{H}, 0,0.23089$ $9,2.327594,0.831058 \backslash \mathrm{H}, 0,-0.75247,1.214465,1.799984 \backslash \mathrm{H}, 0,-2.916655,-1.29$ $8951,-1.344968 \backslash \mathrm{H}, 0,-1.555471,2.407128,0.744019 \backslash \backslash$ Version=IA64L-G03RevD. $01 \backslash$ State $=1-A \backslash H F=-890.4341585 \backslash M P 2=-891.9107639 \backslash$ RMSD $=5.423 e-09 \backslash \mathrm{Thermal}=0$ . $\backslash \mathrm{PG}=\mathrm{C} 01[\mathrm{X}(\mathrm{C} 5 \mathrm{H} 704 \mathrm{~S} 1)] \backslash \backslash \mathrm{Q}$

$1 e$

$1 \backslash 1 \backslash G I N C-B A R O S S A 105 \backslash S P \backslash R O M P 2-F C \backslash 6-31 G(d) \backslash C 5 H 605 \backslash S A N D A L G \backslash 28-J U n-2005 \backslash 0 \backslash$

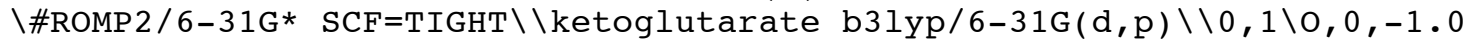
$30302,-1.27474,1.253115 \backslash \mathrm{C}, 0,-1.48686,-0.637806,0.331668 \backslash \mathrm{C}, 0,-1.017619$, $0.787429,-0.048526 \backslash 0,0,-1.668793,1.380063,-0.891199 \backslash 0,0,-2.480075,-1.0$ $59915,-0.451486 \backslash \mathrm{H}, 0,-2.657541,-0.325098,-1.075834 \backslash \mathrm{C}, 0,0.118116,1.42438$ $7,0.722708 \backslash \mathrm{H}, 0,0.383146,2.337671,0.183662 \backslash \mathrm{H}, 0,-0.289782,1.726069,1.696$ $512 \backslash \mathrm{C}, 0,1.347701,0.536734,0.962308 \backslash \mathrm{H}, 0,1.113631,-0.246781,1.689565 \backslash \mathrm{H}, 0$ , $2.178894,1.12231,1.364467 \backslash \mathrm{C}, 0,1.78394,-0.163467,-0.301977 \backslash 0,0,1.06711$ $3,-0.354577,-1.264861 \backslash 0,0,3.058345,-0.593996,-0.243398 \backslash \mathrm{H}, 0,3.229673,-1$ $.072527,-1.072828 \backslash \backslash$ Version=IA32L-G03RevC.02\State $=1-A \backslash H F=-567.1753291 \backslash$ $\mathrm{MP} 2=-568.6965741 \backslash \mathrm{RMSD}=7.545 \mathrm{e}-09 \backslash \mathrm{PG}=\mathrm{C} 01 \quad[\mathrm{X}(\mathrm{C} 5 \mathrm{H} 6 \mathrm{O} 5)] \backslash \backslash @$

\section{$2 e$}

$1 \backslash 1 \backslash G I N C-B A R O S S A 124 \backslash S P \backslash R O M P 2-F C \backslash 6-31 G(d) \backslash C 5 H 5 O 5$ ( 2 ) \SANDALG $\backslash 29-J u n-2005$ $\backslash 0 \backslash \backslash \#$ ROMP2/6-31G* SCF=TIGHT \\ketoglutarate substrate radical b3lyp/6-3 $1 \mathrm{G}(\mathrm{d}, \mathrm{p}) \backslash \backslash 0,2 \backslash \mathrm{O}, 0,-2.332793,-1.236453,-0.325062 \backslash \mathrm{H}, 0,-2.634545,-0.548614$ $,-0.953597 \backslash \mathrm{C}, 0,-1.338271,-0.689542,0.374076 \backslash 0,0,-0.742743,-1.243048,1$. $267881 \backslash \mathrm{C}, 0,-1.056956,0.770546,-0.05215 \backslash 0,0,-1.804298,1.28701,-0.85972 \backslash$ $\mathrm{C}, 0,0.049082,1.507789,0.685866 \backslash \mathrm{H}, 0,0.189819,2.462446,0.160021 \backslash \mathrm{H}, 0,-0.3$ $29828,1.747634,1.687822 \backslash \mathrm{C}, 0,1.328961,0.753818,0.802647 \backslash \mathrm{H}, 0,1.952018,0$. $840729,1.684234 \backslash \mathrm{C}, 0,1.751745,-0.082345,-0.297471 \backslash 0,0,1.060459,-0.29185$ $9,-1.29204 \backslash 0,0,2.979122,-0.625603,-0.133966 \backslash \mathrm{H}, 0,3.137201,-1.184172,-0$. $913045 \backslash \backslash$ Version=IA32L-G03RevC.02 $\backslash$ State $=2-A \backslash H F=-566.54683 \backslash \mathrm{MP} 2=-568.0472$ $184 \backslash \mathrm{RMSD}=8.187 e-09 \backslash \mathrm{PG}=\mathrm{C} 01[\mathrm{X}(\mathrm{C} 5 \mathrm{H} 5 \mathrm{O} 5)] \backslash \backslash \mathrm{a}$

\section{C3-radical derived from $1 \mathrm{e}$}

$1 \backslash 1 \backslash G I N C-A C 7 \backslash S P \backslash R O M P 2-F C \backslash 6-31 G(d) \backslash C 5 H 5 O 5$ ( 2 ) \GMS501\14-Jul-2005

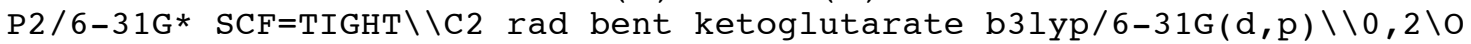
$, 0,0.543721,-1.490185,-0.727425 \backslash \mathrm{C}, 0,1.379622,-0.775096,-0.222853 \backslash \mathrm{C}, 0,1$ $.233728,0.748566,-0.042878 \backslash 0,0,2.22519,1.355113,0.412105 \backslash 0,0,2.5599,-1$ $.210668,0.220563 \backslash \mathrm{H}, 0,3.030923,-0.415325,0.549337 \backslash \mathrm{C}, 0,0.035578,1.432185$ $,-0.404397 \backslash \mathrm{H}, 0,0.081981,2.510591,-0.280135 \backslash \mathrm{C}, 0,-1.238997,0.797285,-0.8$ $43867 \backslash \mathrm{H}, 0,-1.063488,0.079462,-1.652385 \backslash \mathrm{H}, 0,-1.949339,1.546972,-1.20470$ $1 \backslash \mathrm{C}, 0,-1.904945,0.008304,0.283375 \backslash 0,0,-1.473106,-0.122985,1.404212 \backslash 0,0$ $,-3.068289,-0.526388,-0.139408 \backslash \mathrm{H}, 0,-3.429313,-1.02828,0.611229 \backslash \backslash$ Versio $\mathrm{n}=\mathrm{IA} 64 \mathrm{~L}-\mathrm{G} 03 \mathrm{RevC} .02 \backslash \mathrm{State}=2-\mathrm{A} \backslash \mathrm{HF}=-566.5462927 \backslash \mathrm{MP} 2=-568.050455 \backslash \mathrm{RMSD}=2.54$ $1 e-09 \backslash P G=C 01 \quad[X(C 5 H 505)] \backslash \backslash @$ 


\section{TS:2e $\rightarrow 3 e^{*}$}

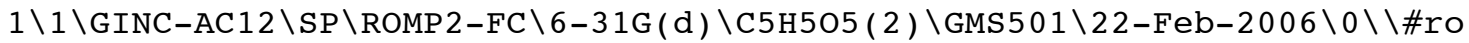
mp2/6-31G* scf=tight \\TS intra ketoglutarate substrate radical b3lyp/6 $-31 \mathrm{G}(\mathrm{d}, \mathrm{p}) \backslash \backslash 0,2 \backslash \mathrm{C}, 0,0.577728,-0.572256,1.520253 \backslash \mathrm{C}, 0,-1.239777,0.737548$, $0.550461 \backslash 0,0,-1.05099,1.817822,0.08778 \backslash \mathrm{C}, 0,-2.147338,-0.336401,-0.1358$ $12 \backslash 0,0,-2.059397,-1.515099,0.069948 \backslash 0,0,-3.104409,0.22243,-0.896735 \backslash \mathrm{H}$, $0,-2.915778,1.171236,-1.00045 \backslash \mathrm{C}, 0,1.184283,-0.88217,0.354944 \backslash \mathrm{C}, 0,2.271$ $401,-0.029685,-0.174346 \backslash 0,0,2.698195,0.981903,0.346084 \backslash 0,0,2.757993,-0$ $.515407,-1.344288 \backslash \mathrm{H}, 0,0.93206,0.26768,2.108884 \backslash \mathrm{H}, 0,-0.175403,-1.225213$ $, 1.944376 \backslash \mathrm{H}, 0,0.885918,-1.743789,-0.23253 \backslash \mathrm{H}, 0,3.464284,0.094676,-1.615$

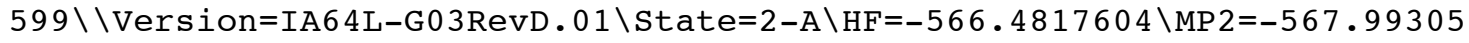
$51 \backslash \mathrm{RMSD}=6.742 \mathrm{e}-09 \backslash \mathrm{Thermal}=0 . \backslash \mathrm{PG}=\mathrm{C} 01 \quad[\mathrm{X}(\mathrm{C} 5 \mathrm{H} 505)] \backslash \backslash \mathrm{a}$

\section{TS: $3 e^{*} \rightarrow 4 e$}

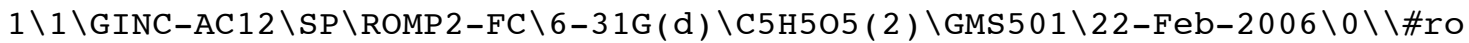
mp2/6-31G* scf=tight \\TS intra ketoglutarate substrat b3lyp/6-31G(d,p) $\backslash \backslash 0,2 \backslash \mathrm{C}, 0,0.902407,0.927959,0.105698 \backslash \mathrm{C}, 0,-0.708343,-0.628674,-0.00302 \backslash$ $0,0,-0.607195,-1.582605,-0.7145 \backslash 0,0,-2.101937,1.273028,0.628985 \backslash \mathrm{C}, 0,-2$ $.034286,0.140677,0.230665 \backslash 0,0,-3.107858,-0.653159,0.061862 \backslash \mathrm{H}, 0,-2.8223$ $95,-1.485728,-0.353151 \backslash \mathrm{C}, 0,0.583168,1.553346,-1.059441 \backslash \mathrm{C}, 0,2.053791,-0$ $.021246,0.143296 \backslash 0,0,2.659537,-0.427727,-0.824049 \backslash 0,0,2.349994,-0.3854$ $4,1.41232 \backslash \mathrm{H}, 0,1.069129,1.258607,-1.983926 \backslash \mathrm{H}, 0,-0.180522,2.321175,-1.08$ $6596 \backslash \mathrm{H}, 0,0.514622,1.28345,1.053624 \backslash \mathrm{H}, 0,3.098418,-1.002656,1.349926 \backslash \backslash \mathrm{Ve}$ rsion=IA6 4L-G03RevD. $01 \backslash$ State $=2-A \backslash H F=-566.4709307 \backslash M P 2=-567.9885714 \backslash$ RMSD $=6.354 \mathrm{e}-09 \backslash \mathrm{Thermal}=0 . \backslash \mathrm{PG}=\mathrm{C} 01 \quad[\mathrm{X}(\mathrm{C} 5 \mathrm{H} 505)] \backslash \backslash \mathrm{a}$

\section{$4 e$}

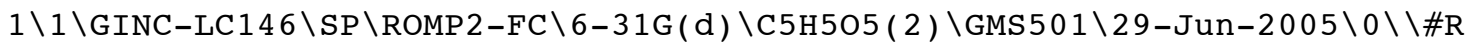
OMP2/6-31G* SCF=TIGHT MAXDISK=72089600\\rotate $\mathrm{COOH}$ ketoglutarate prod

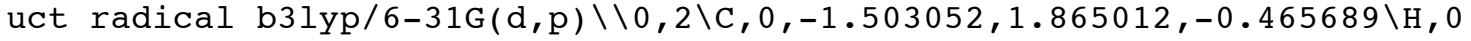
$,-1.121805,2.676605,0.140209 \backslash \mathrm{H}, 0,-2.480654,1.938336,-0.923975 \backslash \mathrm{C}, 0,-0.7$ $48924,0.581121,-0.54464 \backslash \mathrm{H}, 0,-0.72762,0.18343,-1.569104 \backslash \mathrm{C}, 0,0.684645,0$. $694939,-0.053102 \backslash \mathrm{C}, 0,1.587785,-0.536963,-0.285215 \backslash 0,0,1.163487,1.67636$ $2,0.478593 \backslash 0,0,1.216128,-1.510895,-0.893928 \backslash 0,0,2.80853,-0.384117,0.23$ $1598 \backslash \mathrm{H}, 0,2.829351,0.501421,0.646291 \backslash \mathrm{C}, 0,-1.429347,-0.497994,0.316855 \backslash \mathrm{O}$ $, 0,-2.531442,-0.982421,-0.283507 \backslash 0,0,-1.047383,-0.837129,1.413328 \backslash \mathrm{H}, 0$, $-2.920469,-1.630867,0.328655 \backslash \backslash$ Version=IA32L-G03RevC . 02 \State=2-A $\backslash H F=-5$ $66.5333546 \backslash \mathrm{MP} 2=-568.0317895 \backslash \mathrm{RMSD}=7.562 \mathrm{e}-09 \backslash \mathrm{PG}=\mathrm{C} 01 \quad[\mathrm{X}(\mathrm{C} 5 \mathrm{H} 505)] \backslash \backslash \mathrm{a}$

\section{$5 e$}

$1 \backslash 1 \backslash G I N C-L C 9 \backslash S P \backslash R O M P 2-F C \backslash 6-31 G(d) \backslash C 5 H 605 \backslash G M S 501 \backslash 29-J u n-2005 \backslash 0 \backslash \backslash \# R O M P 2 /$ 6-31G* SCF=TIGHT MAXDISK=72089600\\ketoglutarate product b3lyp/6-31G(d , p) $\backslash \backslash 0,1 \backslash \mathrm{C}, 0,-1.531987,1.864389,-0.372924 \backslash \mathrm{C}, 0,-0.747279,0.551375,-0.51$ $5633 \backslash \mathrm{H}, 0,-0.726942,0.223323,-1.561444 \backslash \mathrm{C}, 0,0.689349,0.684115,-0.045956 \backslash$ $\mathrm{C}, 0,1.608929,-0.538144,-0.271524 \backslash 0,0,1.167582,1.683465,0.454164 \backslash 0,0,1$. $243904,-1.529175,-0.856205 \backslash 0,0,2.836165,-0.355067,0.220405 \backslash \mathrm{H}, 0,2.84770$ $8,0.539813,0.615484 \backslash \mathrm{C}, 0,-1.393574,-0.561402,0.300403 \backslash 0,0,-2.442757,-1$. $111332,-0.340309 \backslash 0,0,-1.041824,-0.881626,1.414434 \backslash \mathrm{H}, 0,-1.560407,2.1844$ $28,0.67127 \backslash \mathrm{H}, 0,-1.054047,2.655706,-0.954759 \backslash \mathrm{H}, 0,-2.55432,1.731317,-0.7$ $33087 \backslash \mathrm{H}, 0,-2.809176,-1.786693,0.256425 \backslash \backslash$ Version=IA32L-G03RevC.02\State $=1-\mathrm{A} \backslash \mathrm{HF}=-567.1714878 \backslash \mathrm{MP} 2=-568.693486 \backslash \mathrm{RMSD}=4.941 \mathrm{e}-09 \backslash \mathrm{PG}=\mathrm{C} 01 \quad[\mathrm{X}(\mathrm{C} 5 \mathrm{H} 6 \mathrm{O5})]$ $\backslash \backslash \mathrm{Q}$ 


\section{$3 e^{\prime}$}

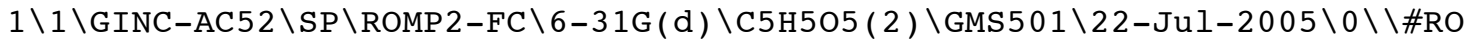
MP2/6-31G* SCF=TIGHT \\TS intra ketoglutarate substrate radical b3lyp/6 $-31 \mathrm{G}(\mathrm{d}, \mathrm{p}) \backslash \backslash 0,2 \backslash \mathrm{C}, 0,0.151325,-0.699915,1.512551 \backslash \mathrm{C}, 0,0.612356,-0.703702$, $0.131091 \backslash \mathrm{C}, 0,1.970332,-0.109336,-0.1445 \backslash 0,0,2.66754,0.440867,0.673925 \backslash$ $\mathrm{O}, 0,2.301941,-0.263139,-1.442552 \backslash \mathrm{O}, 0,-2.798923,0.793221,-0.412722 \backslash \mathrm{H}, 0$, $-2.30828,1.628341,-0.267183 \backslash \mathrm{C}, 0,-1.92062,-0.194906,-0.218189 \backslash 0,0,-2.18$ $6614,-1.371151,-0.311764 \backslash \mathrm{C}, 0,-0.5328,0.342199,0.130111 \backslash 0,0,-0.351466,1$ $.579156,0.21484 \backslash \mathrm{H}, 0,0.653069,-0.055374,2.224036 \backslash \mathrm{H}, 0,-0.60473,-1.402414$ $, 1.837758 \backslash \mathrm{H}, 0,0.346552,-1.567563,-0.474589 \backslash \mathrm{H}, 0,3.170011,0.159338,-1.56$ $0216 \backslash \backslash$ Version=IA64L-G03RevC.02\State=2-A \HF=-566.4965811 \MP2=-568.0249 $365 \backslash \mathrm{RMSD}=4.258 \mathrm{e}-09 \backslash \mathrm{PG}=\mathrm{C} 01 \quad[\mathrm{X}(\mathrm{C} 5 \mathrm{H} 505)] \backslash \backslash \mathrm{a}$

\section{$1 f$}

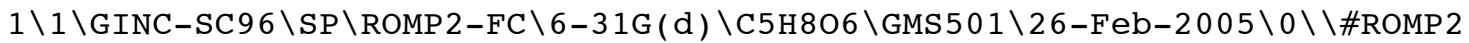
/6-31G* SCF=TIGHT \\hydrate of ketoglutarate romp2/6-31G*//b31yp/6-31G( $\mathrm{d}, \mathrm{p}) \backslash \backslash 0,1 \backslash \mathrm{C}, 0,-0.802516,-0.222059,0.095193 \backslash \mathrm{C}, 0,-2.304559,0.116924,-0.0$ $2771 \backslash 0,0,-3.162923,-0.47182,0.593279 \backslash 0,0,-2.564736,1.093113,-0.91378 \backslash \mathrm{H}$ $, 0,-3.532415,1.185917,-0.949001 \backslash \mathrm{C}, 0,0.08477,1.041917,0.11058 \backslash 0,0,-0.57$ $9297,-1.053931,-1.009742 \backslash 0,0,-0.612899,-0.875284,1.325277 \backslash \mathrm{H}, 0,-0.41590$ $9,1.802944,0.717182 \backslash \mathrm{H}, 0,0.140016,1.432815,-0.90848 \backslash \mathrm{C}, 0,1.484365,0.8465$ $72,0.711078 \backslash \mathrm{C}, 0,2.446818,0.032016,-0.116872 \backslash \mathrm{H}, 0,1.399201,0.344716,1.68$ $1955 \backslash \mathrm{H}, 0,1.953373,1.816389,0.904253 \backslash \mathrm{O}, 0,2.162328,-0.739124,-1.018259 \backslash 0$ $, 0,3.724354,0.227395,0.260372 \backslash \mathrm{H}, 0,4.270196,-0.358902,-0.291561 \backslash \mathrm{H}, 0,0.3$ $88537,-1.116309,-1.137092 \backslash \mathrm{H}, 0,-1.390884,-1.442592,1.451954 \backslash \backslash$ Version=Al $64 \mathrm{~T}-\mathrm{G} 03$ RevC.02 $\backslash \mathrm{State}=1-\mathrm{A} \backslash \mathrm{HF}=-643.2068855 \backslash \mathrm{MP} 2=-644.9160472 \backslash \mathrm{RMSD}=3.219 \mathrm{e}-$ $09 \backslash P G=C 01 \quad[X(C 5 H 806)] \backslash \backslash @$

\section{$2 f$}

$1 \backslash 1 \backslash G I N C-S C 32 \backslash S P \backslash R O M P 2-F C \backslash 6-31 G(d) \backslash C 5 H 706$ ( 2 ) \GMS501\26-Feb-2005 $0 \backslash \backslash \# R O$ MP 2/6-31G* SCF=TIGHT \\hydrate of ketoglutarate romp $2 / 6-31 \mathrm{G} * / / \mathrm{b} 31 \mathrm{yp} / 6-3$ $1 \mathrm{G}(\mathrm{d}, \mathrm{p}) \backslash \backslash 0,2 \backslash \mathrm{C}, 0,-0.772771,0.206236,0.118174 \backslash \mathrm{C}, 0,-2.284201,-0.049514,-$ $0.069406 \backslash 0,0,-3.115953,0.818676,0.078434 \backslash 0,0,-2.579316,-1.324848,-0.37$ $0891 \backslash \mathrm{H}, 0,-3.549277,-1.385168,-0.414371 \backslash \mathrm{C}, 0,0.085297,-0.525469,-0.95964$ $5 \backslash 0,0,-0.527389,-0.250095,1.414361 \backslash \mathrm{O}, 0,-0.539111,1.579131,-0.049457 \backslash \mathrm{H}$, $0,-0.410553,-0.426168,-1.927995 \backslash \mathrm{H}, 0,0.086454,-1.587552,-0.690516 \backslash \mathrm{C}, 0,1$ $.475444,0.000132,-1.074968 \backslash \mathrm{C}, 0,2.442927,-0.139105,-0.010334 \backslash \mathrm{H}, 0,1.7883$ $12,0.535212,-1.964112 \backslash 0,0,2.201854,-0.574585,1.118219 \backslash 0,0,3.686012,0.2$ $63589,-0.358995 \backslash \mathrm{H}, 0,4.242023,0.147268,0.429396 \backslash \mathrm{H}, 0,0.440499,-0.366039$, $1.518272 \backslash \mathrm{H}, 0,-1.286413,2.033822,0.373034 \backslash \backslash$ Version=Al64T-G03RevC.02\Sta $\mathrm{te}=2-\mathrm{A} \backslash \mathrm{HF}=-642.5792009 \backslash \mathrm{MP} 2=-644.26761 \backslash \mathrm{RMSD}=7.705 \mathrm{e}-09 \backslash \mathrm{PG}=\mathrm{C} 01 \quad[\mathrm{X}(\mathrm{C} 5 \mathrm{H} 706)$ ]$\backslash \backslash$ Q

\section{C3-radical derived from $1 f$}

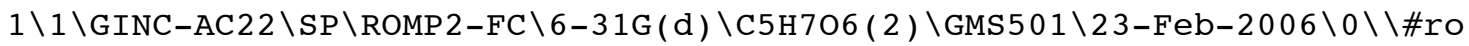

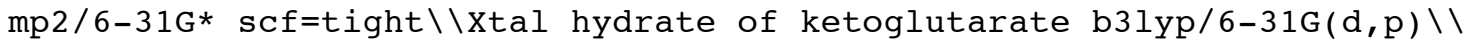
$0,2 \backslash 0,0,1.399795,-1.645085,0.785773 \backslash \mathrm{C}, 0,0.912039,-0.50466,0.137175 \backslash \mathrm{C}, 0$ $, 2.113842,0.455834,-0.091348 \backslash 0,0,3.267,0.091963,-0.027601 \backslash \mathrm{C}, 0,-0.15332$ $4,0.083365,1.014755 \backslash \mathrm{C}, 0,-1.27476,0.901214,0.462821 \backslash \mathrm{C}, 0,-2.423558,0.043$ $369,-0.059416 \backslash 0,0,-2.301068,-1.013046,-0.651704 \backslash 0,0,-3.626824,0.593912$ $, 0.183349 \backslash 0,0,1.74383,1.712871,-0.392134 \backslash \mathrm{H}, 0,2.561415,2.21306,-0.55850$ $3 \backslash 0,0,0.458701,-0.746701,-1.176939 \backslash \mathrm{H}, 0,-0.128523,-0.177209,2.066837 \backslash \mathrm{H}$, $0,-0.92532,1.490099,-0.395492 \backslash \mathrm{H}, 0,-4.290189,-0.000233,-0.209498 \backslash \mathrm{H}, 0,-1$ $.671644,1.597183,1.206041 \backslash \mathrm{H}, 0,2.300169,-1.786021,0.448208 \backslash \mathrm{H}, 0,-0.42281$ $1,-1.16293,-1.107461 \backslash \backslash$ Version=IA64L-G03RevD. $01 \backslash$ State=2-A $\backslash H F=-642.57172$ $66 \backslash \mathrm{MP} 2=-644.257867 \backslash \mathrm{RMSD}=7.899 \mathrm{e}-09 \backslash \mathrm{Thermal}=0 . \backslash \mathrm{PG}=\mathrm{C} 01 \quad[\mathrm{X}(\mathrm{C} 5 \mathrm{H} 706)] \backslash \backslash @$ 


\section{TS:2f $\rightarrow 3 f^{*}$}

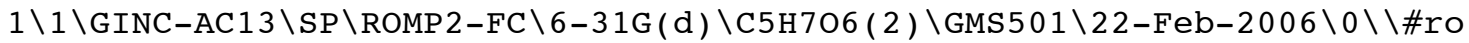

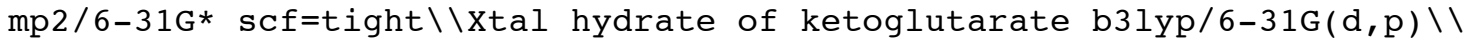
$0,2 \backslash \mathrm{C}, 0,0.260126,0.011755,1.385336 \backslash \mathrm{C}, 0,-1.083842,0.630401,-0.220786 \backslash 0$, $0,-0.272262,0.789412,-1.263183 \backslash \mathrm{H}, 0,0.594644,1.178987,-0.985186 \backslash 0,0,-1$. $631756,1.751864,0.307403 \backslash \mathrm{H}, 0,-2.419775,1.451548,0.800905 \backslash 0,0,-3.0014,-$ $0.532356,0.504739 \backslash \mathrm{C}, 0,-1.992121,-0.539142,-0.19052 \backslash 0,0,-1.570779,-1.59$ $6111,-0.901243 \backslash \mathrm{H}, 0,-2.221737,-2.301698,-0.749654 \backslash \mathrm{C}, 0,1.305541,-0.73051$ $6,0.872895 \backslash \mathrm{C}, 0,2.337283,-0.070438,0.095791 \backslash 0,0,2.251667,1.071315,-0.37$ $4566 \backslash \mathrm{O}, 0,3.443576,-0.818203,-0.099262 \backslash \mathrm{H}, 0,0.395194,1.08094,1.515247 \backslash \mathrm{H}$, $0,-0.454789,-0.456175,2.055279 \backslash \mathrm{H}, 0,1.354899,-1.809017,0.969398 \backslash \mathrm{H}, 0,4.0$ $37276,-0.284313,-0.653394 \backslash \backslash$ Version=IA64L-G03RevD . $01 \backslash$ State $=2-A \backslash H F=-642$. $5157941 \backslash \mathrm{MP} 2=-644.2290304 \backslash \mathrm{RMSD}=2.547 \mathrm{e}-09 \backslash \mathrm{Therma}=0 . \backslash \mathrm{PG}=\mathrm{C} 01[\mathrm{X}(\mathrm{C} 5 \mathrm{H} 7 \mathrm{O6})] \backslash @$

\section{TS: $3 f^{*} \rightarrow 4 f$}

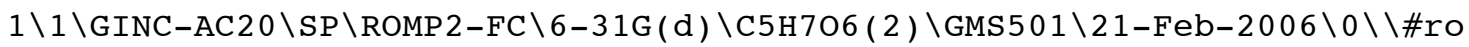
mp2/6-31G* scf=tight \\radical from 24 hydketo_prod1 hydrate of ketoglu

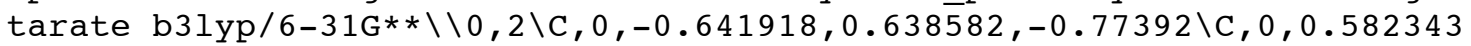
$,-0.611811,0.342938 \backslash 0,0,0.394598,-1.81965,-0.204654 \backslash \mathrm{H}, 0,-0.578493,-1.9$ $59705,-0.304761 \backslash \mathrm{O}, 0,0.192899,-0.486486,1.643489 \backslash \mathrm{H}, 0,0.677456,0.284687$, $1.991967 \backslash \mathrm{C}, 0,1.88655,0.057763,0.067329 \backslash 0,0,2.437733,-0.259247,-1.11370$ $5 \backslash \mathrm{H}, 0,3.237319,0.287388,-1.197701 \backslash \mathrm{O}, 0,2.332119,0.898884,0.830796 \backslash \mathrm{C}, 0,-$ $1.889179,-0.010417,-0.301889 \backslash 0,0,-2.693873,0.779526,0.430135 \backslash 0,0,-2.17$ $2911,-1.188854,-0.50674 \backslash \mathrm{C}, 0,-0.428886,2.008444,-0.550387 \backslash \mathrm{H}, 0,-0.276918$ $, 0.2045,-1.70162 \backslash \mathrm{H}, 0,0.352025,2.537804,-1.083487 \backslash \mathrm{H}, 0,-0.965749,2.54331$ $2,0.222766 \backslash \mathrm{H}, 0,-3.423626,0.213249,0.733836 \backslash \backslash$ Version=IA64L-G03RevD.01\S tate $=2-\mathrm{A} \backslash \mathrm{HF}=-642.5113856 \backslash \mathrm{MP} 2=-644.2226512 \backslash \mathrm{RMSD}=2.431 \mathrm{e}-09 \backslash \mathrm{Thermal}=0 . \backslash \mathrm{PG}$ $=\mathrm{C} 01[\mathrm{X}(\mathrm{C} 5 \mathrm{H} 706)] \backslash \backslash \mathrm{a}$

\section{$4 f$}

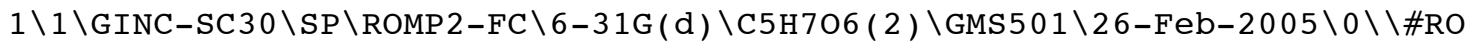
MP2/6-31G* SCF=TIGHT \radical from 24 hydketo_prod1 hydrate of ketoglu tarate romp2/6-31G*//b3lyp/6-31G**\\0,2\C,0,0.675409,-0.755356,0.46127 $4 \backslash \mathrm{C}, 0,1.762453,0.183738,-0.036557 \backslash 0,0,1.63503,1.390889,-0.192305 \backslash 0,0,2$ $.928838,-0.432668,-0.244729 \backslash \mathrm{H}, 0,3.565017,0.247306,-0.529483 \backslash \mathrm{C}, 0,-0.656$ $224,-0.573139,-0.341226 \backslash \mathrm{C}, 0,0.503656,-0.582252,1.938049 \backslash \mathrm{H}, 0,0.984089,-$ $1.778634,0.234575 \backslash \mathrm{H}, 0,0.46519,0.41044,2.372485 \backslash \mathrm{H}, 0,0.302326,-1.439666$, $2.566624 \backslash 0,0,-1.43515,-1.703893,-0.14931 \backslash 0,0,-0.272712,-0.426732,-1.69$ $9674 \backslash \mathrm{C}, 0,-1.526328,0.658466,0.075888 \backslash 0,0,-2.709372,0.480747,0.30593 \backslash 0$, $0,-0.964624,1.852221,0.105637 \backslash \mathrm{H}, 0,0.00886,1.79836,-0.088545 \backslash \mathrm{H}, 0,-1.013$ $645,-0.755705,-2.229094 \backslash \mathrm{H}, 0,-2.32172,-1.355365,0.084475 \backslash \backslash \operatorname{Version}=\mathrm{Al} 64 \mathrm{~T}$ $-\mathrm{G} 03$ RevC.02 2 State $=2-\mathrm{A} \backslash \mathrm{HF}=-642.5615826 \backslash \mathrm{MP} 2=-644.2534107 \backslash \mathrm{RMSD}=6.747 e-09 \backslash$ $\mathrm{PG}=\mathrm{C} 01[\mathrm{X}(\mathrm{C} 5 \mathrm{H} 7 \mathrm{O})] \backslash \backslash @$

\section{$5 f$}

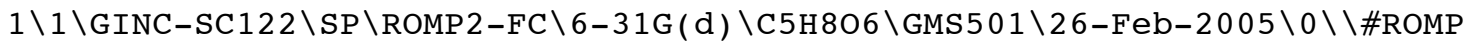
2/6-31G* SCF=TIGHT \\24 hydketo_prod1 hydrate of ketoglutarate romp2/6$31 \mathrm{G} * / / \mathrm{b} 31 \mathrm{yp} / 6-31 \mathrm{G} * * \backslash \backslash 0,1 \backslash \mathrm{C}, 0,0.681033,-0.800791,0.268351 \backslash \mathrm{C}, 0,1.752503$, $0.216976,-0.06362 \backslash 0,0,1.610805,1.433202,-0.055544 \backslash 0,0,2.939894,-0.3434$ $09,-0.318443 \backslash \mathrm{H}, 0,3.568786,0.381433,-0.483049 \backslash \mathrm{C}, 0,-0.663905,-0.504236,-$ $0.436598 \backslash \mathrm{C}, 0,0.528581,-0.929977,1.795734 \backslash \mathrm{H}, 0,1.002436,-1.761028,-0.143$ $126 \backslash \mathrm{H}, 0,0.256958,0.026758,2.251103 \backslash \mathrm{H}, 0,1.460048,-1.276669,2.250094 \backslash \mathrm{H}, 0$ $,-0.255548,-1.658909,2.011431 \backslash 0,0,-1.44001,-1.656033,-0.380946 \backslash 0,0,-0$. $333249,-0.155921,-1.773017 \backslash \mathrm{C}, 0,-1.528708,0.65012,0.176185 \backslash 0,0,-2.7083$, $0.436564,0.396044 \backslash \mathrm{O}, 0,-0.968851,1.828328,0.373446 \backslash \mathrm{H}, 0,0.001096,1.80688$ $5,0.150166 \backslash \mathrm{H}, 0,-1.126356,-0.309629,-2.306561 \backslash \mathrm{H}, 0,-2.326754,-1.343241,-$ $0.102688 \backslash \backslash$ Version=Al64T-G03RevC . 02 \State $=1-\mathrm{A} \backslash \mathrm{HF}=-643.1994194 \backslash \mathrm{MP} 2=-644$. $9158228 \backslash \mathrm{RMSD}=4.219 \mathrm{e}-09 \backslash \mathrm{PG}=\mathrm{C} 01 \quad[\mathrm{X}(\mathrm{C} 5 \mathrm{H} 806)] \backslash \backslash \mathrm{a}$ 


\section{1s}

$1 \backslash 1 \backslash G I N C-A C 16 \backslash S P \backslash R O M P 2-F C \backslash 6-31 G(d) \backslash C 5 H 10 N 204 \backslash G M S 501 \backslash 18-S e p-2005 \backslash 0 \backslash \backslash \# R$

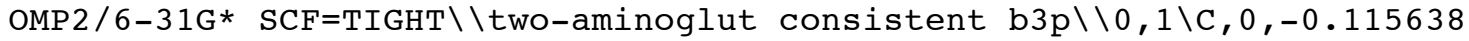
$,-0.754035,-0.650489 \backslash \mathrm{C}, 0,-1.506084,-1.024288,-0.062236 \backslash \mathrm{C}, 0,-2.477954,0$ $.124065,-0.205903 \backslash \mathrm{C}, 0,0.88122,-0.087569,0.319521 \backslash 0,0,-2.204436,1.27085$ $,-0.509601 \backslash 0,0,-3.745804,-0.259339,0.053916 \backslash \mathrm{N}, 0,1.016281,-0.917146,1.5$ $26897 \backslash \mathrm{N}, 0,0.501752,1.270368,0.750461 \backslash \mathrm{C}, 0,2.267685,0.005645,-0.391767 \backslash 0$ $, 0,2.855391,1.199182,-0.274553 \backslash 0,0,2.776811,-0.948805,-0.939695 \backslash \mathrm{H}, 0,-1$ $.968451,-1.899343,-0.530097 \backslash \mathrm{H}, 0,-4.299681,0.533874,-0.045293 \backslash \mathrm{H}, 0,0.114$ $413,1.225855,1.689857 \backslash \mathrm{H}, 0,-0.237607,1.637272,0.151412 \backslash \mathrm{H}, 0,0.33782,-1.6$ $94281,-0.975274 \backslash \mathrm{H}, 0,-0.200762,-0.129111,-1.545879 \backslash \mathrm{H}, 0,-1.423492,-1.263$ $435,1.005662 \backslash \mathrm{H}, 0,1.416658,-1.813311,1.252632 \backslash \mathrm{H}, 0,1.704199,-0.489397,2$. $14713 \backslash \mathrm{H}, 0,2.179601,1.741316,0.21306 \backslash \backslash$ Version=IA64L-G03RevC.02\State=1$\mathrm{A} \backslash \mathrm{HF}=-603.535457 \backslash \mathrm{MP} 2=-605.2233003 \backslash \mathrm{RMSD}=5.221 \mathrm{e}-09 \backslash \mathrm{PG}=\mathrm{C} 01 \quad[\mathrm{X}(\mathrm{C} 5 \mathrm{H} 10 \mathrm{~N} 2 \mathrm{O} 4)]$ $\backslash \backslash Q$

\section{2s}

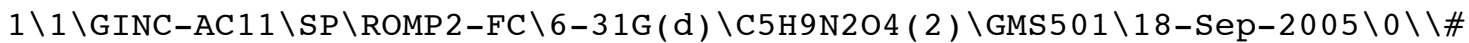

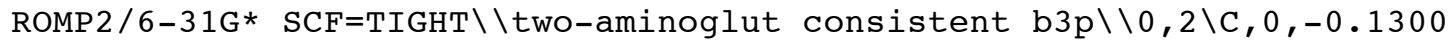
$63,-0.737886,-0.702044 \backslash \mathrm{C}, 0,-1.49548,-0.969388,-0.166116 \backslash \mathrm{C}, 0,-2.490157$, $0.079904,-0.228776 \backslash \mathrm{C}, 0,0.857682,-0.100211,0.321392 \backslash 0,0,-2.271823,1.229$ $324,-0.610383 \backslash 0,0,-3.718821,-0.315604,0.188343 \backslash \mathrm{N}, 0,0.962646,-0.960561$, $1.505859 \backslash \mathrm{N}, 0,0.463754,1.240014,0.776888 \backslash \mathrm{C}, 0,2.253741,0.010519,-0.36867$ $8 \backslash 0,0,2.863265,1.181508,-0.16298 \backslash 0,0,2.752009,-0.915942,-0.969941 \backslash \mathrm{H}, 0$, $-4.295511,0.462005,0.107212 \backslash \mathrm{H}, 0,-0.004843,1.15393,1.675767 \backslash \mathrm{H}, 0,-0.2182$ $61,1.647925,0.136931 \backslash \mathrm{H}, 0,0.323171,-1.672258,-1.045337 \backslash \mathrm{H}, 0,-0.183585,-0$ $.060176,-1.561309 \backslash \mathrm{H}, 0,-1.76198,-1.901541,0.318406 \backslash \mathrm{H}, 0,1.334935,-1.8637$ $1,1.216559 \backslash \mathrm{H}, 0,1.658813,-0.566613,2.13927 \backslash \mathrm{H}, 0,2.191079,1.712349,0.3382$ $79 \backslash \backslash$ Version=IA6 4L-G03RevC. 02 \State $=2-A \backslash H F=-602.9097783 \backslash M P 2=-604.576313$ $\backslash \mathrm{RMSD}=4.054 \mathrm{e}-09 \backslash \mathrm{PG}=\mathrm{C} 01 \quad[\mathrm{X}(\mathrm{C} 5 \mathrm{H} 9 \mathrm{~N} 2 \mathrm{O} 4)] \backslash \backslash \mathrm{a}$

\section{TS:2s $\rightarrow 3 s^{*}$}

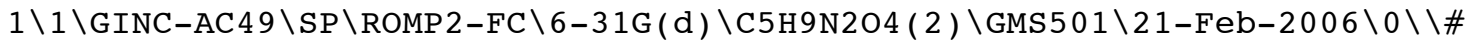
romp2/6-31G* scf=tight \\two-aminoglut Xtal $\mathrm{COOH} b 3 \mathrm{p} \backslash \backslash 0,2 \backslash \mathrm{C}, 0,0.182605$ $, 0.208054,1.314029 \backslash \mathrm{C}, 0,-1.158626,0.64167,-0.194458 \backslash \mathrm{N}, 0,-0.432288,0.938$ $574,-1.350011 \backslash \mathrm{H}, 0,-0.059907,0.098036,-1.776417 \backslash \mathrm{H}, 0,0.342536,1.57305,-1$ $.161668 \backslash \mathrm{N}, 0,-1.846227,1.73218,0.359327 \backslash \mathrm{H}, 0,-2.63818,1.424845,0.914272 \backslash$ $\mathrm{H}, 0,-2.120501,2.404923,-0.348447 \backslash \mathrm{C}, 0,-1.88247,-0.659967,-0.153083 \backslash 0,0$, $-1.297039,-1.612978,-0.918786 \backslash \mathrm{H}, 0,-1.801241,-2.430321,-0.772418 \backslash 0,0,-2$ $.855366,-0.873126,0.54796 \backslash \mathrm{C}, 0,1.238884,-0.601936,0.891638 \backslash \mathrm{C}, 0,2.337598$ $,-0.027082,0.142743 \backslash 0,0,2.396494,1.127024,-0.283125 \backslash 0,0,3.35453,-0.906$ $687,-0.076692 \backslash \mathrm{H}, 0,0.379198,1.271738,1.40332 \backslash \mathrm{H}, 0,-0.510268,-0.189432,2$. $051474 \backslash \mathrm{H}, 0,1.239068,-1.675554,1.043106 \backslash \mathrm{H}, 0,4.022006,-0.410856,-0.57849$ $4 \backslash \backslash$ Version=IA6 4L-G03RevD. $01 \backslash$ State $=2-A \backslash H F=-602.8609156 \backslash M P 2=-604.5503894$ $\backslash \mathrm{RMSD}=8.466 \mathrm{e}-09 \backslash \mathrm{Thermal}=0 . \backslash \mathrm{PG}=\mathrm{C} 01 \quad[\mathrm{X}(\mathrm{C} 5 \mathrm{H} 9 \mathrm{~N} 204)] \backslash \backslash @$

\section{TS: $3 s^{*} \rightarrow 4 s$}

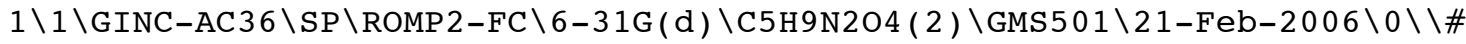
romp2/6-31G* scf=tight \\two-aminoglut Xtal $\mathrm{COOH} b 3 \mathrm{p} \backslash \backslash 0,2 \backslash \mathrm{H}, 0,0.914414$ $,-2.077118,-0.930899 \backslash \mathrm{C}, 0,-0.614506,0.628602,-0.735664 \backslash \mathrm{C}, 0,0.574898,-0$. $589867,0.39523 \backslash \mathrm{N}, 0,0.071542,-0.405593,1.704828 \backslash \mathrm{H}, 0,0.491359,-1.075117$, $2.345371 \backslash \mathrm{H}, 0,0.265108,0.537523,2.027447 \backslash \mathrm{N}, 0,0.471514,-1.917883,-0.0342$ $66 \backslash \mathrm{H}, 0,-0.493157,-2.23555,-0.040358 \backslash 0,0,2.39304,-0.345229,-1.117841 \backslash \mathrm{C}$, $0,1.868928,0.083392,0.063115 \backslash \mathrm{H}, 0,3.191324,0.187594,-1.269497 \backslash 0,0,2.360$ $839,0.982092,0.713611 \backslash \mathrm{C}, 0,-1.943615,0.18733,-0.221489 \backslash 0,0,-2.67477,0.8$ $1348,0.513085 \backslash 0,0,-2.28125,-1.06384,-0.665871 \backslash \mathrm{C}, 0,-0.335491,2.00285,-0$ $.581685 \backslash \mathrm{H}, 0,-0.3146,0.153807,-1.668294 \backslash \mathrm{H}, 0,0.488255,2.470651,-1.106752$ 
$\backslash \mathrm{H}, 0,-0.884832,2.588842,0.145315 \backslash \mathrm{H}, 0,-3.143412,-1.252164,-0.257177 \backslash \backslash \mathrm{Ve}$ rsion=IA64L-G03RevD. $01 \backslash$ State $=2-A \backslash H F=-602.8565296 \backslash M P 2=-604.538541 \backslash \mathrm{RMSD}=$ $9.167 e-09 \backslash \mathrm{Thermal}=0 . \backslash \mathrm{PG}=\mathrm{C} 01 \quad[\mathrm{X}(\mathrm{C} 5 \mathrm{H} 9 \mathrm{~N} 204)] \backslash \backslash @$

\section{4s}

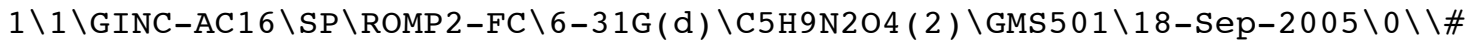

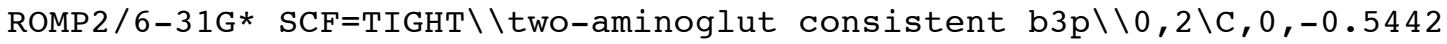
$41,0.496662,-0.444156 \backslash \mathrm{C}, 0,0.536364,-0.461812,0.22458 \backslash \mathrm{N}, 0,0.392572,-1.8$ $60742,-0.12075 \backslash \mathrm{H}, 0,-0.562238,-2.187887,-0.006607 \backslash \mathrm{H}, 0,0.703804,-2.0166$, $-1.076016 \backslash \mathrm{C}, 0,-0.399108,1.938021,-0.076954 \backslash \mathrm{C}, 0,-1.950375,0.046233,-0.0$ $71641 \backslash 0,0,-2.317059,-0.254885,1.049743 \backslash 0,0,-2.784765,0.042872,-1.12877$ $9 \backslash \mathrm{N}, 0,0.558743,-0.248843,1.67571 \backslash \mathrm{C}, 0,1.902266,0.001311,-0.35803 \backslash 0,0,2$. $257051,-0.295079,-1.477309 \backslash 0,0,2.59849,0.780444,0.468633 \backslash \mathrm{H}, 0,-0.416515$ $, 0.374395,-1.522304 \backslash \mathrm{H}, 0,-3.664153,-0.200961,-0.789696 \backslash \mathrm{H}, 0,2.05396,0.80$ $1639,1.296363 \backslash \mathrm{H}, 0,-0.391759,-0.131624,2.022569 \backslash \mathrm{H}, 0,0.930741,-1.098061$, $2.098216 \backslash \mathrm{H}, 0,0.077081,2.633314,-0.756603 \backslash \mathrm{H}, 0,-0.689288,2.283574,0.9082$ $59 \backslash \backslash$ Version=IA6 4L-G03RevC . 02 \State=2-A \HF=-602.8966263\MP2=-604.564477 $2 \backslash \mathrm{RMSD}=3.663 \mathrm{e}-09 \backslash \mathrm{PG}=\mathrm{C} 01 \quad[\mathrm{X}(\mathrm{C} 5 \mathrm{H} 9 \mathrm{~N} 2 \mathrm{O} 4)] \backslash \backslash @$

\section{5s}

$1 \backslash 1 \backslash G I N C-A C 11 \backslash S P \backslash R O M P 2-F C \backslash 6-31 G(d) \backslash C 5 H 10 N 204 \backslash G M S 501 \backslash 18-S e p-2005 \backslash 0 \backslash \backslash \# R$

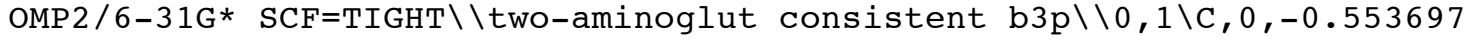
$, 0.552196,-0.247018 \backslash \mathrm{C}, 0,0.551467,-0.519842,0.035811 \backslash \mathrm{N}, 0,0.455237,-1.71$ $6514,-0.787477 \backslash \mathrm{H}, 0,-0.490396,-2.087864,-0.785617 \backslash \mathrm{H}, 0,0.740051,-1.50118$ $5,-1.739578 \backslash \mathrm{C}, 0,-0.441246,1.839111,0.602781 \backslash \mathrm{C}, 0,-1.947027,-0.025619,-0$ $.072783 \backslash 0,0,-2.258635,-0.927867,0.683218 \backslash 0,0,-2.85518,0.608893,-0.8421$ $66 \backslash \mathrm{N}, 0,0.597264,-0.853033,1.467327 \backslash \mathrm{C}, 0,1.904864,0.153115,-0.339391 \backslash 0,0$ $, 2.247818,0.312673,-1.48923 \backslash 0,0,2.609083,0.569492,0.715371 \backslash \mathrm{H}, 0,-0.4438$ $58,0.829338,-1.300206 \backslash \mathrm{H}, 0,-3.720737,0.216471,-0.632863 \backslash \mathrm{H}, 0,2.075797,0$. $271013,1.495724 \backslash \mathrm{H}, 0,-0.352115,-0.953878,1.821032 \backslash \mathrm{H}, 0,1.028945,-1.77383$ $9,1.538291 \backslash \mathrm{H}, 0,-1.206613,2.558374,0.300822 \backslash \mathrm{H}, 0,0.536618,2.305178,0.462$ $231 \backslash \mathrm{H}, 0,-0.566048,1.623933,1.66728 \backslash \backslash$ Version=IA64L-G03RevC .02 $\backslash$ State=1-A $\backslash \mathrm{HF}=-603.5345056 \backslash \mathrm{MP} 2=-605.2255805 \backslash \mathrm{RMSD}=5.249 \mathrm{e}-09 \backslash \mathrm{PG}=\mathrm{C} 01 \quad[\mathrm{X}(\mathrm{C} 5 \mathrm{H} 10 \mathrm{~N} 2 \mathrm{O} 4)]$ $\backslash \backslash \mathrm{Q}$

\section{Tetrahydro-5-methylfuran-3,4-diol}

$1 \backslash 1 \backslash G I N C-A C 17 \backslash S P \backslash R O M P 2-F C \backslash 6-31 G(d) \backslash C 5 H 1003 \backslash G M S 501 \backslash 19-O c t-2005 \backslash 0 \backslash \backslash \#$ ROM

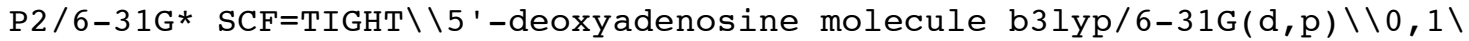
$0,0,0.612579,-1.23887,-0.598293 \backslash \mathrm{C}, 0,1.009655,0.149528,-0.446837 \backslash \mathrm{C}, 0,-0$ $.066469,0.811322,0.472128 \backslash \mathrm{C}, 0,-1.136214,-0.302405,0.590076 \backslash \mathrm{C}, 0,-0.2781$ $57,-1.551706,0.476988 \backslash \mathrm{H}, 0,0.953324,0.607787,-1.440696 \backslash \mathrm{H}, 0,0.36562,1.02$ $2705,1.458089 \backslash \mathrm{H}, 0,-1.733438,-0.237247,1.502583 \backslash \mathrm{H}, 0,-0.843796,-2.447372$ $, 0.206498 \backslash \mathrm{H}, 0,0.26477,-1.744836,1.415783 \backslash \mathrm{C}, 0,2.432436,0.245149,0.08802$ $\backslash \mathrm{H}, 0,2.735988,1.29439,0.169233 \backslash \mathrm{H}, 0,3.131815,-0.268387,-0.577205 \backslash \mathrm{H}, 0,2$. $510162,-0.209289,1.081995 \backslash 0,0,-2.045487,-0.19437,-0.509022 \backslash \mathrm{H}, 0,-1.5839$ $73,-0.567128,-1.276995 \backslash 0,0,-0.592877,2.018909,-0.025505 \backslash \mathrm{H}, 0,-1.361695$, $1.752695,-0.558969 \backslash \backslash$ Version=IA64L-G03RevC.02 $\backslash$ State $=1-A \backslash H F=-419.7152713$ $\backslash \mathrm{MP} 2=-420.903483 \backslash \mathrm{RMSD}=9.710 \mathrm{e}-09 \backslash \mathrm{PG}=\mathrm{C} 01 \quad[\mathrm{X}(\mathrm{C} 5 \mathrm{H} 1003)] \backslash \backslash @$

\section{Tetrahydro-5-methylfuran-3,4-diol radical}

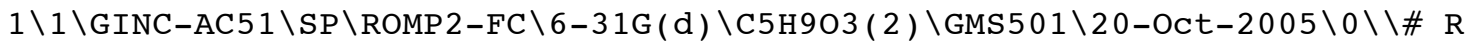

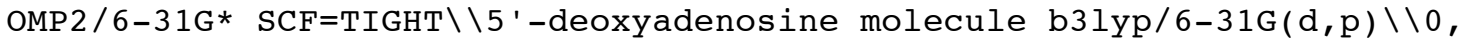
$2 \backslash 0,0,0.704577,-1.148106,-0.664716 \backslash \mathrm{C}, 0,1.059722,0.240116,-0.441669 \backslash \mathrm{C}, 0$ $,-0.082571,0.843742,0.461459 \backslash \mathrm{C}, 0,-1.038515,-0.369579,0.626295 \backslash \mathrm{C}, 0,-0.0$ $83299,-1.541505,0.463736 \backslash \mathrm{H}, 0,1.010698,0.738716,-1.422591 \backslash \mathrm{H}, 0,0.332513$, $1.137285,1.431885 \backslash \mathrm{H}, 0,-1.595832,-0.356915,1.565692 \backslash \mathrm{H}, 0,-0.582991,-2.48$ $3664,0.225081 \backslash \mathrm{H}, 0,0.538416,-1.67698,1.362271 \backslash \mathrm{C}, 0,2.420349,0.350182,0.1$ 
$46949 \backslash \mathrm{H}, 0,2.773767,1.305337,0.521922 \backslash \mathrm{H}, 0,3.128205,-0.461541,0.02332 \backslash 0$, $0,-2.003342,-0.349024,-0.429445 \backslash \mathrm{H}, 0,-1.540587,-0.677406,-1.217382 \backslash 0,0$, $-0.728673,1.964176,-0.089524 \backslash \mathrm{H}, 0,-1.498803,1.601058,-0.56134 \backslash \backslash$ Version $=$ IA64L-G03RevC.02\State $=2-A \backslash H F=-419.077168 \backslash M P 2=-420.2425664 \backslash \mathrm{RMSD}=7.189 \mathrm{e}$ $-09 \backslash P G=C 01 \quad[X(C 5 H 903)] \backslash \backslash @$ 


\section{G3(MP2)-RAD//B3-LYP/6-31G(d,p) Total Energies (Hartrees) at $0 \mathrm{~K}$}

\begin{tabular}{|c|c|}
\hline Species & Energy \\
\hline $\mathbf{1 a}$ & -550.89286 \\
\hline $\mathbf{2 a}$ & -550.24423 \\
\hline C3-radical derived from $1 a$ & -550.23289 \\
\hline Acrylic acid & -266.80264 \\
\hline TS:2a $\rightarrow 3 \mathbf{a}^{*}$ & -550.21768 \\
\hline $\mathrm{TS}: 3 \mathbf{a}^{*} \rightarrow \mathbf{4 a}$ & -550.21570 \\
\hline $4 \mathbf{a}$ & -550.23331 \\
\hline $\mathbf{5 a}$ & -550.89481 \\
\hline $1 \mathbf{b}$ & -570.76300 \\
\hline $2 b$ & -570.11309 \\
\hline C3-radical derived from $\mathbf{1 b}$ & -570.10170 \\
\hline $\mathrm{TS}: 2 \mathrm{~b} \rightarrow 3 \mathrm{~b}^{*}$ & -570.07996 \\
\hline $\mathrm{TS}: 3 b^{*} \rightarrow 4 b$ & -570.07644 \\
\hline $4 b$ & -570.10206 \\
\hline $5 \mathbf{b}$ & -570.76468 \\
\hline $\mathrm{H}-\mathrm{HC}\left(\mathrm{CO}_{2} \mathrm{H}\right)-\mathrm{H}$ & -228.78251 \\
\hline $\mathrm{H}-\cdot \mathrm{C}\left(\mathrm{CO}_{2} \mathrm{H}\right)-\mathrm{H}$ & -228.12559 \\
\hline $\mathrm{H}-\mathrm{HC}\left(\mathrm{CO}_{2} \mathrm{H}\right)-\mathrm{NH}_{2}$ & -284.05429 \\
\hline $\mathrm{H}-\bullet \mathrm{C}\left(\mathrm{CO}_{2} \mathrm{H}\right)-\mathrm{NH}_{2}(3 \mathrm{a})$ & -283.42520 \\
\hline $\mathrm{H}-\mathrm{HC}\left(\mathrm{CO}_{2} \mathrm{H}\right)-\mathrm{SH}$ & -625.53324 \\
\hline $\mathrm{H}-\cdot \mathrm{C}\left(\mathrm{CO}_{2} \mathrm{H}\right)-\mathrm{SH}(3 \mathrm{c})$ & -625.89922 \\
\hline $\mathrm{H}-\mathrm{HC}\left(\mathrm{CO}_{2} \mathrm{H}\right)-\mathrm{S}^{-}$ & -626.00569 \\
\hline $\mathrm{H}-\cdot \mathbf{C}\left(\mathrm{CO}_{2} \mathrm{H}\right)-\mathbf{S}^{-}(\mathbf{3 d})$ & -625.38884 \\
\hline $\mathrm{H}-\mathrm{HC}\left(\mathrm{CO}_{2} \mathrm{H}\right)-\mathrm{OH}$ & -303.92173 \\
\hline $\mathrm{H}-\bullet \mathrm{C}\left(\mathrm{CO}_{2} \mathrm{H}\right)-\mathrm{OH}(3 \mathrm{~b})$ & -303.28623 \\
\hline
\end{tabular}




\begin{tabular}{|c|c|}
\hline $\mathrm{H}-\mathrm{HC}\left(\mathrm{CO}_{2} \mathrm{H}\right)-\mathrm{O}^{-}$ & -303.38913 \\
\hline $\mathbf{H}-\cdot \mathbf{C}\left(\mathrm{CO}_{2} \mathrm{H}\right)-\mathbf{O}^{-}$ & -302.75587 \\
\hline $\mathrm{H}-\mathrm{HC}\left(\mathrm{CO}_{2} \mathrm{H}\right)-\mathrm{F}$ & -327.93119 \\
\hline $\mathrm{H}-\cdot \mathbf{C}\left(\mathrm{CO}_{2} \mathrm{H}\right)-\mathrm{F}$ & -327.28255 \\
\hline $\mathrm{HO}-\mathrm{HC}\left(\mathrm{CO}_{2} \mathrm{H}\right)-\mathrm{NH}_{2}$ & -359.20574 \\
\hline $\mathrm{HO}-\cdot \mathrm{C}\left(\mathrm{CO}_{2} \mathrm{H}\right)-\mathrm{NH}_{2}$ & -358.57986 \\
\hline $\mathrm{H}_{2} \mathrm{~N}-\mathrm{HC}\left(\mathrm{CO}_{2} \mathrm{H}\right)-\mathrm{NH}_{2}$ & -339.33471 \\
\hline $\mathrm{H}_{2} \mathrm{~N}-\cdot \mathrm{C}\left(\mathrm{CO}_{2} \mathrm{H}\right)-\mathrm{NH}_{2}(3 \mathrm{~s})$ & -338.71341 \\
\hline $\mathrm{F}-\mathrm{HC}\left(\mathrm{CO}_{2} \mathrm{H}\right)-\mathrm{NH}_{2}$ & -383.22059 \\
\hline $\mathrm{F}-\cdot \mathrm{C}\left(\mathrm{CO}_{2} \mathrm{H}\right)-\mathrm{NH}_{2}$ & -382.58038 \\
\hline $\mathrm{HO}-\mathrm{HC}\left(\mathrm{CO}_{2} \mathrm{H}\right)-\mathrm{OH}$ & -379.07336 \\
\hline $\mathrm{HO}-\cdot C\left(\mathrm{CO}_{2} \mathrm{H}\right)-\mathrm{OH}(3 \mathrm{f})$ & -378.44154 \\
\hline $\mathrm{O}=\mathrm{HC}\left(\mathrm{CO}_{2} \mathrm{H}\right)$ & -302.71673 \\
\hline $\mathrm{O}=\cdot \mathrm{C}\left(\mathrm{CO}_{2} \mathrm{H}\right)(3 \mathrm{e})$ & -302.07118 \\
\hline 1c & -893.37477 \\
\hline $2 c$ & -892.72400 \\
\hline C3-radical derived from 1c & -892.71671 \\
\hline $\mathrm{TS}: 2 \mathrm{c} \rightarrow 3 \mathrm{c}^{*}$ & -892.69179 \\
\hline $\mathrm{TS}: 3 \mathrm{c} * \mathbf{4 c}$ & -892.68685 \\
\hline $4 c$ & -892.71068 \\
\hline $5 c$ & -893.37281 \\
\hline $1 d$ & -892.83179 \\
\hline $2 d$ & -892.18547 \\
\hline C3-radical derived from 1d & -892.17755 \\
\hline $\mathbf{T S}: \mathbf{2 d} \rightarrow \mathbf{3 d} *$ & -892.17629 \\
\hline $\mathrm{TS}: 3 \mathrm{~d} * \rightarrow \mathbf{4 d}$ & -892.15740 \\
\hline $4 d$ & -892.17174 \\
\hline $5 d$ & -892.83335 \\
\hline
\end{tabular}




\begin{tabular}{|c|c|}
\hline 1e & -569.56684 \\
\hline $2 e$ & -568.91588 \\
\hline C3-radical derived from $1 e$ & -568.91789 \\
\hline $\mathrm{TS}: 2 \mathrm{e} \rightarrow 3 \mathrm{e}^{*}$ & -568.86684 \\
\hline $\mathrm{TS}: 3 \mathrm{e}^{*} \rightarrow 4 \mathrm{e}$ & -568.86375 \\
\hline $4 e$ & -568.90205 \\
\hline $5 e$ & -569.56473 \\
\hline $3 \mathbf{e}^{\prime}$ & -568.88922 \\
\hline 1f & -645.92179 \\
\hline $2 f$ & -645.27083 \\
\hline C3-radical derived from $1 f$ & -645.26136 \\
\hline $\mathrm{TS}: 2 \mathrm{f} \rightarrow 3 \mathrm{f}^{*}$ & -645.23853 \\
\hline $\mathrm{TS}: 3 f^{*} \rightarrow \mathbf{4 f}$ & -645.23516 \\
\hline $4 \mathbf{f}$ & -645.26025 \\
\hline $5 f$ & -645.92313 \\
\hline 1s & -606.17731 \\
\hline $2 s$ & -605.52800 \\
\hline $\mathrm{TS}: 2 \mathrm{~s} \rightarrow 3 \mathrm{~s}^{*}$ & -605.50751 \\
\hline $\mathrm{TS}: 3 \mathrm{~s}^{*} \rightarrow 4 \mathrm{~s}$ & -605.49869 \\
\hline $4 s$ & -605.51747 \\
\hline $5 \mathrm{~s}$ & -606.17948 \\
\hline Tetrahydro -5-methylfuran-3,4-diol & -421.58926 \\
\hline Tetrahydro -5-methylfuran-3,4-diol radical & -420.92686 \\
\hline
\end{tabular}




\section{FULL REFERENCES}

(23) MOLPRO 2002.6 is a package of ab initio programs written by Werner, H.-J.; Knowles, P. J.; Schütz, M.; Lindh, R.; Celani, P.; Korona, T.; Rauhut, G.; Manby, F. R.; Amos, R. D.; Bernhardsson, A.; Berning, A. Cooper, D. L.; Deegan, M. J. O.; Dobbyn, A. J.; Eckert, F.; Hampel, C.; Hetzer, G.; Lloyd, A. W.; McNicholas, S. J.; Meyer, W.; Mura, M. E.; Nicklaß, A.; Palmieri, P; Pitzer, R.; Schumann, U.; Stoll, H.; Stone, A. J.; Tarroni, R.; Thorsteinsson, T.

(24) Frisch, M. J.; Trucks, G. W.; Schlegel, H. B.; Scuseria, G. E.; Robb, M. A.; Cheeseman, J. R.; Montgomery, J. A., Jr.; Vreven, T.; Kudin, K. N.; Burant, J. C.; Millam, J. M.; Iyengar, S. S.; Tomasi, J.; Barone, V.; Mennucci, B.; Cossi, M.; Scalmani, G.; Rega, N.; Petersson, G. A.; Nakatsuji, H.; Hada, M.; Ehara, M.; Toyota, K.; Fukuda, R.; Hasegawa, J.; Ishida, M.; Nakajima, T.; Honda, Y.; Kitao, O.; Nakai, H.; Klene, M.; Li, X.; Knox, J. E.; Hratchian, H. P.; Cross, J. B.; Adamo, C.; Jaramillo, J.; Gomperts, R.; Stratmann, R. E.; Yazyev, O.; Austin, A. J.; Cammi, R.; Pomelli, C.; Ochterski, J. W.; Ayala, P. Y.; Morokuma, K.; Voth, G. A.; Salvador, P.; Dannenberg, J. J.; Zakrzewski, V. G.; Dapprich, S.; Daniels, A. D.; Strain, M. C.; Farkas, O.; Malick, D. K.; Rabuck, A. D.; Raghavachari, K.; Foresman, J. B.; Ortiz, J. V.; Cui, Q.; Baboul, A. G.; Clifford, S.; Cioslowski, J.; Stefanov, B. B.; Liu, G.; Liashenko, A.; Piskorz, P.; Komaromi, I.; Martin, R. L.; Fox, D. J.; Keith, T.; Al-Laham, M. A.; Peng, C. Y.; Nanayakkara, A.; Challacombe, M.; Gill, P. M. W.; Johnson, B.; Chen, W.; Wong, M. W.; Gonzalez, C.; Pople, J. A., Gaussian, Inc., Pittsburgh, PA, 2003. 